\title{
Review Article \\ Systematic Review of Adverse Effects: A Further Step towards Modernization of Acupuncture in China
}

\author{
Junyi Wu, ${ }^{1}$ Yanmei Hu, ${ }^{1}$ Yin Zhu, ${ }^{2}$ Ping Yin, ${ }^{1}$ Gerhard Litscher, ${ }^{3}$ and Shifen $\mathrm{Xu}^{1}$ \\ ${ }^{1}$ Acupuncture Department, Shanghai Municipal Hospital of Traditional Chinese Medicine, Shanghai 200071, China \\ ${ }^{2}$ Huadong Hospital Affiliated to Fudan University, Shanghai 200040, China \\ ${ }^{3}$ Research Unit for Complementary and Integrative Laser Medicine, Research Unit of Biomedical Engineering in Anesthesia and \\ Intensive Care Medicine, and TCM Research Center Graz, Medical University of Graz, 8036 Graz, Austria
}

Correspondence should be addressed to Shifen Xu; xu_teacher2006@126.com

Received 8 July 2014; Accepted 30 July 2014

Academic Editor: Lu Wang

Copyright (C) 2015 Junyi Wu et al. This is an open access article distributed under the Creative Commons Attribution License, which permits unrestricted use, distribution, and reproduction in any medium, provided the original work is properly cited.

As a further step towards the modernization of acupuncture, the objective of this review was to figure out the frequency and severity of adverse complications and events in acupuncture treatment reported from 1980 to 2013 in China. All first-hand case reports of acupuncture-related complications and adverse events that could be identified in the scientific literature were reviewed and classified according to the type of complication and adverse event, circumstance of the event, and long-term patient outcome. The selected case reports were published between 1980 and 2013 in 3 databases. Relevant papers were collected and analyzed by 2 reviewers. Over the 33 years, 182 incidents were identified in 133 relevant papers. Internal organ, tissue, or nerve injury is the main complications of acupuncture especially for pneumothorax and central nervous system injury. Adverse effects also included syncope, infections, hemorrhage, allergy, burn, aphonia, hysteria, cough, thirst, fever, somnolence, and broken needles. Qualifying training of acupuncturists should be systemized and the clinical acupuncture operations should be standardized in order to effectively prevent the occurrence of acupuncture accidents, enhance the influence of acupuncture, and further popularize acupuncture to the rest of the world.

\section{Introduction}

Complications and adverse effects in medical practice are always of concern to the public and the medical profession. While being widely used in current medical treatment, especially in the area of intertrochanteric hip fractures, the cephalomedullary devices (CMN), however, led to a considerably high complication rate of $41.9 \%$ according to Pui et al. [1]. Faunø et al. [2] conducted a study on the medical records of 997 patients who were suffering from stoma closure, which revealed 131 cases of early complications and 187 late ones, accounting for $13.1 \%$ and $18.8 \%$ of the total subject pool, respectively. Umuroglu et al. [3] identified that the nuss procedure showed an overall complication rate of $18.7 \%$ through a retrospective analysis. Fortunately, with the increasing attention from the society on medical care safety and the further standardization of doctor's qualifications, such medical incidents have been decreasing. As acupuncture and moxibustion (A\&M) are increasingly used in world, their widening acceptance necessitates continual safety assessment. This review, a sequel to two previous reviews from our team $[4,5]$, is an evaluation of the frequency and severity of adverse events (AEs) for acupuncture reported between 1980 and 2013 in China. These two reviews reported the AEs of A\&M in the West, reported from 1965 to 1999 and from 2000 to 2011, respectively. In the first review, the main source of infection was found to be hepatitis caused by reused needles. In the second review, the majority of infections were bacterial, caused by skin contact at acupoint sites, without cases of hepatitis. For these two reviews, we just searched English articles, without Chinese reports, so it is unclear which AE happens in China. Since acupuncture is widely used in China, it is necessary to make sure what is happening about AE. In this review, we found internal organ, tissue, or 
nerve injuries were the main complications of acupuncture in China from 1980 to 2013. Clearly, guidelines must be followed in order to minimize acupuncture-related AEs and to modernize acupuncture and make it safer to apply.

\section{Materials and Methods}

2.1. Inclusion Criteria. All case reports concerning acupuncture-related complications or AEs in China published from 1980 to 2013 were identified. Only firsthand reports were included to avoid multiple reports of the same event. Thus, reviews, comments, or case-control studies were excluded from this review.

Complications, defined as "an added difficulty: a complex state, a disease, or an accident superimposed upon another without specific relation," include infections, internal organ or tissue injuries, and other severe consequences. AEs and adverse reactions were defined as "development of an undesired side effect or toxicity" and include less severe consequences, such as contact dermatitis. Side effects, defined as "the action or effect other than that desired," are included within the discussion of AEs $[4,5]$.

2.2. Search Strategy. The following databases were searched for case reports published from 1980 to 2013: VIP science and technology periodical database (CQVIP), China National Knowledge Infrastructure (CNKI), and Wanfang Database (WF). Search terms included "acupuncture, electro-acupuncture, acupuncture points, ear acupuncture, needling." These terms were combined with "safe, safety, adverse event, adverse reaction, side effects, side events, complications, and risk."

2.3. Data Extraction. A total of 739 papers were found; 133 were relevant (Figure 1). Information pertaining to the author, year of publication, number of patients, patient's age, sex, original treatment, the punctured site, circumstances of the complication or AE, and long-term patient outcome when available was extracted from each case. These data were then organized according to the type of complication or AE.

\section{Results}

A total of 133 papers published from 1980 to 2013 were reviewed, and 182 cases of complications or AEs were identified to be associated with pneumothorax $(n=30)$, central nervous system injury (37), peripheral nerve injury (8), organ injury (22), other tissue injury (18), syncope (18), infections (17), hemorrhage (10), complications caused by broken needles (7), and others (15). Pneumothorax and central nervous system injury were the major complications.

3.1. Complications of Acupuncture: Internal Organ, Tissue, or Nerve Injury. A total of 115 cases of internal organ, tissue, or nerve injuries were reported, including pneumothorax $(n=30)$, central nervous system injury (37), peripheral nerve injury (8), organ injury (22), and other tissue injury (18).
3.2. Pneumothorax (Table 1). Between 1980 and 2013, pneumothorax was the most common complication of acupuncture treatment, as 30 cases in 27 papers were noted to be possibly associated with acupuncture (Table 1). Among the 30 cases presented, 25 recovered through thoracocentesis, thoracic closed drainage, anti-infection treatment, and clinical observation; 2 patients died; and the outcomes of the other 3 cases were not stated or unknown. More than half of them were reported by doctors in emergency rooms or departments of internal medicine, but the practitioner's training background was not reported. The punctured sites were mostly in the chest, supraclavicular fossa, and the back. The patients' major complaints were chest stuffy, chest pain, and dyspnea. One pneumothorax patient did not receive timely treatment and died [6]. Another case died because of a tension pneumothorax after acupuncture treatment [7].

3.3. Central Nervous System Injury (Table 2). There were 37 cases of central nervous system injury associated with acupuncture (Table 2). The 37 patients suffered subarachnoid hemorrhage $(n=27)$, subdural hematoma (2), spinal cord injury (2), cerebral hemorrhage reformulation (2), cervical spinal epidural hematoma (1), medulla oblongata hemorrhage (1), cisterna magna hemorrhage (1), and leukemia acute intracerebral hemorrhage (1). The causes were acupuncture of cervical acupoints $(n=31)$, acupoints between the second and third thoracic spinal process [8], acupoints KI01 (Yongquan) [9] and BL37 (Yinmen) [10], waist acupoint [11] (each $n=1)$, and body acupuncture $(n=2)[12,13]$. Among the 37 cases, 26 recovered and 11 died.

Because of insufficient compliance and protected observation, accidents occurred in 3 schizophrenia patients, of whom 2 died and 1 recovered [14]. Two cerebral hemorrhage patients after receiving body acupuncture died from recurrence, and the authors speculated the reason might be that acupuncture had irritated the parasympathetic nerve, causing vascular contraction and increasing blood pressure and thus cerebral hemorrhage recurred before the original cerebrovascular fracture could be fully restored $[9,12]$. Two patients suffered from dizziness and vomiting during treatment, but the acupuncturists did not pay high attention; the symptoms did not improve significantly after treatment, but the acupuncturists did not realize the severity and even allowed the patients to go home. One patient was treated the next day after onset and was diagnosed as subarachnoid hemorrhage; he recovered and left hospital [11]. The other patient died the next day [13].

3.4. Peripheral Nerve Injury (Table 3). There were 8 cases of acupuncture-induced peripheral nerve injury (Table 3 ). They include aggravated facial paralysis [15], mistakenly hitting the vagus [16], phrenic nerve injury [17], optic atrophy [18], oculomotorius injury [19], right trigeminal nerve injury [20], sciatic nerve injury [10], and peroneus nerve injury [21], respectively. The patient with optic atrophy lost sight [18], the patient with right trigeminal anchor injury improved after 3 days [20], the patient with sciatic nerve injury did not recover [10], and the other 5 patients recovered. 


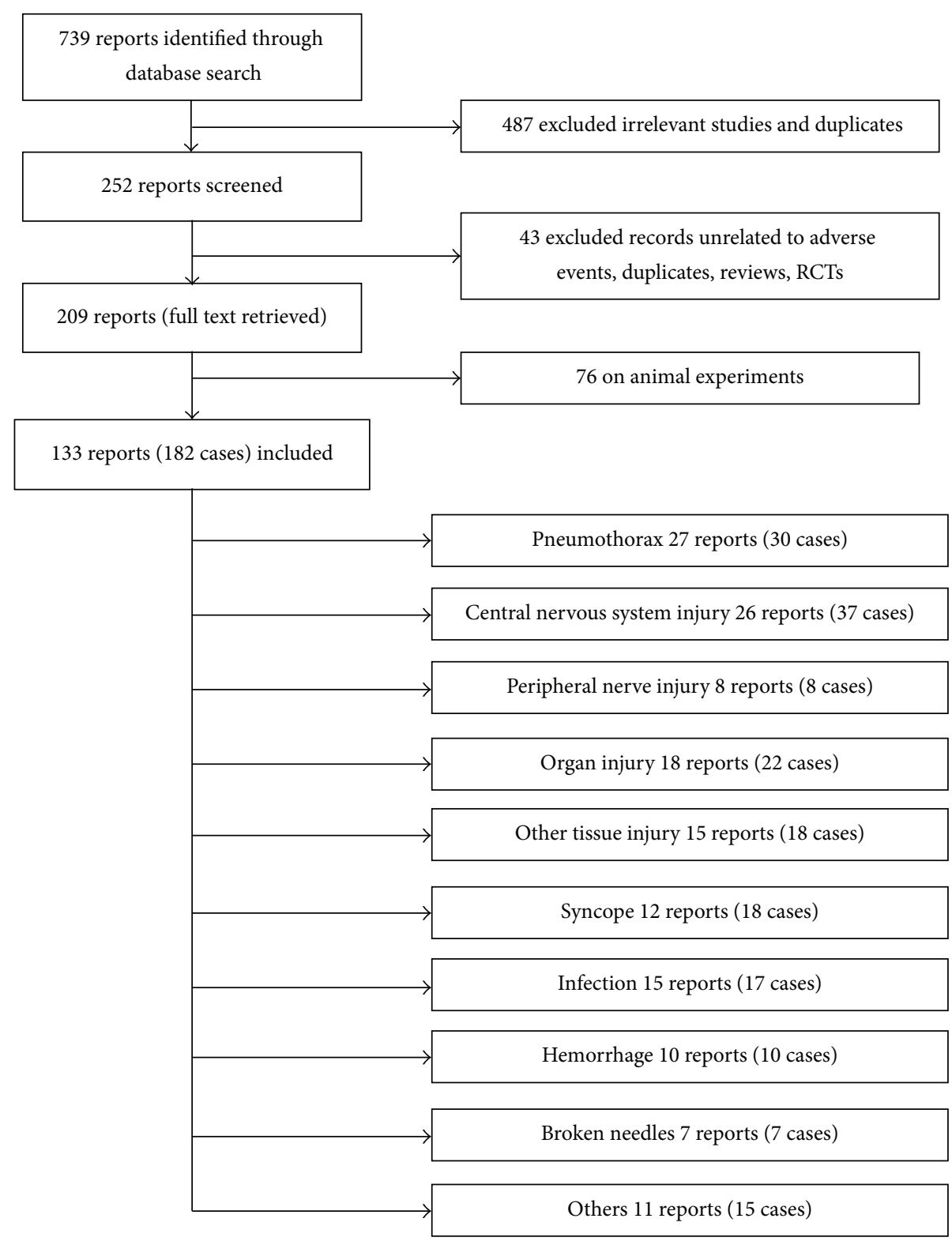

FIGURE 1: Flow chart of the screening process.

3.5. Organ Injury (Table 4). Twenty-two cases were reported to have organ injury associated with acupuncture treatment (Table 4$)$. There was cardiovascular injury $(n=7)[14,22-$ 27], thoracic duct injury (1) [28], peritonitis induced by abdominal system (9) [29-33], gastric perforation (3) [3436], intestinal obstruction (1) [37], and multiple organ injury (1) [38]. Of them, 14 recovered, 7 died, and 1 did not recover. Most of them were caused by too deep puncture and incorrect acupoint location.

One patient received treatment following gastric abscess induced by gastric ulcer. He was treated by electroacupuncture (EA) at ST36 (Zusanli) and the acupoint was located without violation, but the state of illness was not relieved and gastric perforation occurred. The doctor was puzzled and put forward this case for discussion [35]. One patient with a history of stomach bleeding received treatment following knee pains induced by rheumatoid arthritis and took antiinflammatory analgesic drugs for a long time. The lower limb acupoint was selected, but the excessive EA irritation during treatment caused an irritable gastric ulcer and then hemorrhagic shock and the patient recovered after timely rescue [36].

3.6. Other Tissue Injuries (Table 5). In addition to injuries of the organs in thorax and abdomen, 18 cases of other tissue injuries were reported (Table 5), including cervical common carotid aneurysm [39], shock [38, 40], asphyxia [41], dyspnea [42], eye injury $[18,43,44]$, and the locomotor system injury [10, 44-50]. Among the 18 cases, 12 recovered, 2 improved, 2 did not recover, 1 died, and the outcome of 1 was not stated. 
TABLE 1: Pneumothorax associated with acupuncture.

\begin{tabular}{|c|c|c|c|c|c|c|}
\hline Author/year (reference) & Cases & Age/sex & Disease treated & Punctured site & Practitioner & Follow-up \\
\hline Jiang, 1980 [117] & 1 & $54 / \mathrm{F}$ & Gastroptosis & RN15 & Not specified & Recovered \\
\hline Cai and Wang, 1982 [118] & 1 & $41 / \mathrm{M}$ & $\begin{array}{l}\text { Numbness and pain } \\
\text { of shoulders and chest }\end{array}$ & Shoulder and back & Not specified & Recovered (1 mo) \\
\hline Zheng and Pang, 1983 [119] & 1 & $21 / \mathrm{M}$ & Stiff neck & GB21 & Not specified & $\begin{array}{l}\text { Recovered after } \\
\text { surgery }(1 \mathrm{mo})\end{array}$ \\
\hline Gao, $1984[120]$ & 1 & $50 / \mathrm{F}$ & Chest and back pain & Back & Factory doctor & Recovered (12 d) \\
\hline \multirow{2}{*}{ Duan and Wang, 1984 [29] } & \multirow[t]{2}{*}{2} & $26 / \mathrm{F}$ & Bronchitis & GB21, BL13, EX-B2 & Not specified & Recovered (3 d) \\
\hline & & $50 / \mathrm{F}$ & Bronchitis & GB21, BL13 & Not specified & Recovered (7 d) \\
\hline Chang, 1984 [121] & 1 & $33 / \mathrm{M}$ & Back pain & Back & Country doctor & Recovered (19 d) \\
\hline Yan, 1985 [122] & 1 & $55 / \mathrm{F}$ & Chronic bronchitis & $\mathrm{RN} 22$ & Health center & Recovered (16 d) \\
\hline $\mathrm{Hu}, 1986[123]$ & 1 & $58 / \mathrm{F}$ & $\begin{array}{c}\text { Pulmonary heart } \\
\text { disease }\end{array}$ & BL13 & Not specified & Recovered (13 d) \\
\hline Zhang, 1986 [124] & 1 & $52 / \mathrm{M}$ & Cervical pain & Left shoulder & Factory doctor & Recovered (20 d) \\
\hline Yang, 1986 [6] & 1 & $57 / \mathrm{F}$ & $\begin{array}{c}\text { Chronic asthmatic } \\
\text { disease }\end{array}$ & BL12 & Not specified & Dead \\
\hline Jin, 1987 [125] & 1 & $26 / \mathrm{F}$ & Shoulder pain & SI13 & Acupuncturist & Recovered (9d) \\
\hline Song and Xu, 1987 [126] & 1 & $60 / \mathrm{F}$ & Shoulder pain & GB21 & Not specified & Not specified \\
\hline Ruan et al., 1992 [127] & 1 & $35 / \mathrm{F}$ & Hysteria & $\begin{array}{l}\text { Supraclavicular } \\
\text { fossa }\end{array}$ & Acupuncturist & Recovered (10 d) \\
\hline \multirow{3}{*}{ Zhang et al., 1992 [38] } & \multirow{3}{*}{3} & $53 / \mathrm{F}$ & $\begin{array}{l}\text { Chronic bronchitis } \\
\text { and emphysema }\end{array}$ & BL18 & \multirow{3}{*}{ Not specified } & Recovered (1 wk) \\
\hline & & $65 / \mathrm{F}$ & Cough & RN22 & & Recovered (1 mo) \\
\hline & & $21 / \mathrm{M}$ & Spasmodic torticollis & LI17 & & Recovered (2 wk) \\
\hline Lu, 1993 [128] & 1 & $60 / \mathrm{M}$ & $\begin{array}{l}\text { Cough, chest, and } \\
\text { back pain }\end{array}$ & Back & Acupuncturist & Recovered (1 wk) \\
\hline Xia, 1993 [129] & 1 & $60 / \mathrm{M}$ & $\begin{array}{c}\text { Back pain caused by } \\
\text { hyperplastic } \\
\text { spondylitis }\end{array}$ & BL18, BL23 & Acupuncturist & Recovered (10 d) \\
\hline Li and Chen, 1997 [130] & 1 & $65 / F$ & Shoulder pain & Scapular region & Clinic & Recovered (5d) \\
\hline Ma and Zhang, 1997 [131] & 1 & $48 / \mathrm{M}$ & $\begin{array}{l}\text { Neck and shoulder } \\
\text { pain }\end{array}$ & Shoulder and back & Clinic & Recovered (1 wk) \\
\hline Wang, 1999 [7] & 1 & $47 / \mathrm{F}$ & Shoulder and leg pain & LI17 & Not specified & Dead \\
\hline Song and $\mathrm{Wu}, 2001$ [9] & 1 & $45 / \mathrm{F}$ & $\begin{array}{l}\text { Scapulohumeral } \\
\text { periarthritis }\end{array}$ & GB21, SI11 & Hospital & Recovered (1 wk) \\
\hline Qin and Ao, 2003 [132] & 1 & $55 / \mathrm{M}$ & Intercostal neuralgia & $\begin{array}{l}\text { The sixth } \\
\text { intercostal space } \\
\text { on the anterior } \\
\text { axillary line }\end{array}$ & Factory doctor & Recovered (2 wk) \\
\hline Zha, 2006 [133] & 1 & $32 / \mathrm{M}$ & Chronic hepatitis B & Chest and back & Itinerant doctor & Recovered (14 d) \\
\hline Gan et al., 2006 [134] & 1 & $30 / \mathrm{M}$ & Neck and back pain & Back & Individual clinic & Not specified \\
\hline Huo et al., 2007 [135] & 1 & $39 / \mathrm{M}$ & Chest and back pain & Not specified & Not specified & $\begin{array}{l}\text { Recovered after } \\
\text { surgery }(7 \mathrm{~d})\end{array}$ \\
\hline Liu, 2007 [136] & 1 & $50 / \mathrm{M}$ & $\begin{array}{l}\text { Cervical and back } \\
\text { pain }\end{array}$ & Back & Individual clinic & Not specified \\
\hline Ma, 2007 [137] & 1 & $35 / \mathrm{F}$ & Shoulder pain & Not specified & Individual clinic & Recovered \\
\hline Zhang and Zhao, 2012 [138] & 1 & $65 / F$ & $\begin{array}{c}\text { Cervical } \\
\text { spondylopathy }\end{array}$ & GB21 & Acupuncturist & Recovered (10 d) \\
\hline
\end{tabular}


TABLE 2: Central nervous system injury associated with acupuncture.

\begin{tabular}{|c|c|c|c|c|c|c|c|}
\hline Author/Year (reference) & Cases & Age/Sex & Disease treated & Punctured site & Complication & $\begin{array}{c}\text { Onset after } \\
\text { acupuncture }\end{array}$ & Follow-up \\
\hline \multirow[t]{2}{*}{ Liu, 1980 [139] } & 1 & $19 / \mathrm{F}$ & Impaired vision & GB20 & $\begin{array}{l}\text { Subarachnoid } \\
\text { hemorrhage }\end{array}$ & $1 \mathrm{~h}$ & $\begin{array}{l}\text { Recovered } \\
\quad(20 \mathrm{~d})\end{array}$ \\
\hline & & $16 / \mathrm{M}$ & & DU16 & $\begin{array}{c}\text { Subdural } \\
\text { hematoma }\end{array}$ & & \\
\hline \multirow[t]{3}{*}{ Liu, $1981[14]$} & 3 & $30 / \mathrm{F}$ & Schizophrenia & DU14 & $\begin{array}{l}\text { Cervical and } \\
\text { thoracic cord } \\
\text { injury }\end{array}$ & Immediately & Dead \\
\hline & & $30 / \mathrm{M}$ & & GB20 & $\begin{array}{l}\text { Subarachnoid } \\
\text { hemorrhage }\end{array}$ & $1 \mathrm{~h}$ & $\begin{array}{l}\text { Recovered } \\
\quad(47 \mathrm{~d})\end{array}$ \\
\hline & & $30 / \mathrm{F}$ & $\begin{array}{l}\text { Eyelid muscle } \\
\text { twitch }\end{array}$ & GB20 & & & $\begin{array}{l}\text { Recovered } \\
\quad(14 \mathrm{~d})\end{array}$ \\
\hline \multirow[t]{2}{*}{ Bao and Gao, 1983 [140] } & 3 & $27 / \mathrm{M}$ & Bulbar palsy & DU15 & $\begin{array}{l}\text { Subarachnoid } \\
\text { hemorrhage }\end{array}$ & Immediately & $\begin{array}{l}\text { Recovered } \\
\quad(20 \mathrm{~d})\end{array}$ \\
\hline & & $40 / \mathrm{F}$ & Headache & GB20 & & & $\begin{array}{l}\text { Recovered } \\
\quad(19 \mathrm{~d})\end{array}$ \\
\hline Chen and Wu, 1985 [141] & 1 & $30 / \mathrm{F}$ & Hysteria & Ex-HN18 & $\begin{array}{c}\text { Subarachnoid } \\
\text { hemorrhage and } \\
\text { medulla oblongata } \\
\text { hemorrhage }\end{array}$ & $1 \mathrm{~d}$ & Dead \\
\hline Yang et al., 1985 [8] & 1 & $15 / \mathrm{M}$ & Chronic tracheitis & $\begin{array}{c}\text { Between } 2 \text { and } 3 \\
\text { thoracic spinous } \\
\text { process }\end{array}$ & $\begin{array}{l}\text { Subarachnoid } \\
\text { hemorrhage }\end{array}$ & Immediately & $\begin{array}{l}\text { Recovered } \\
\quad(1 \mathrm{mo})\end{array}$ \\
\hline $\begin{array}{l}\text { Chen and Huang, } 1985 \\
{[142]}\end{array}$ & 1 & $57 / \mathrm{M}$ & Facial Spasm & Neck & $\begin{array}{l}\text { Subarachnoid } \\
\text { hemorrhage }\end{array}$ & Immediately & $\begin{array}{l}\text { Recovered } \\
\quad(3 \mathrm{wk})\end{array}$ \\
\hline Su et al., 1985 [143] & 1 & $11 / \mathrm{F}$ & Deaf-mute & DU15 & $\begin{array}{l}\text { Subarachnoid } \\
\text { hemorrhage }\end{array}$ & At night & $\begin{array}{l}\text { Recovered } \\
\quad(1 \mathrm{wk})\end{array}$ \\
\hline \multirow{7}{*}{ Yu, 1986 [144] } & \multirow{7}{*}{7} & $42 / \mathrm{M}$ & Psychosis & DU15 & \multirow{7}{*}{$\begin{array}{l}\text { Subarachnoid } \\
\text { hemorrhage }\end{array}$} & Several hours & $\begin{array}{l}\text { Recovered } \\
\quad(1 \mathrm{mo})\end{array}$ \\
\hline & & $4.5 / \mathrm{M}$ & $\begin{array}{l}\text { Cerebral agenesis } \\
\text { with aphasia }\end{array}$ & DU15 & & Immediately & $\begin{array}{l}\text { Recovered } \\
\quad(20 \mathrm{~d})\end{array}$ \\
\hline & & $29 / \mathrm{M}$ & Weakness of limbs & DU15, DU16 & & $\begin{array}{l}\text { Minutes after } \\
\text { treatment }\end{array}$ & $\begin{array}{l}\text { Recovered } \\
\quad(1 \mathrm{mo})\end{array}$ \\
\hline & & $22 / \mathrm{M}$ & $\begin{array}{l}\text { Acid swells of the } \\
\text { neck }\end{array}$ & Back neck & & \multirow{4}{*}{ Immediately } & $\begin{array}{l}\text { Recovered } \\
\quad(40 \mathrm{~d})\end{array}$ \\
\hline & & $55 / \mathrm{F}$ & Aural vertigo & Back neck & & & $\begin{array}{l}\text { Recovered } \\
\quad(20 \mathrm{~d})\end{array}$ \\
\hline & & $24 / \mathrm{F}$ & Stuffy head & GB20 & & & $\begin{array}{l}\text { Recovered } \\
\quad(1 \mathrm{mo})\end{array}$ \\
\hline & & $22 / \mathrm{M}$ & Facial paralysis & Ex-HN21 & & & Recovered \\
\hline Chen, 1987 [145] & 1 & $37 / \mathrm{F}$ & Neck pain & DU15 & $\begin{array}{l}\text { Subarachnoid } \\
\text { hemorrhage }\end{array}$ & $1 \mathrm{~min}$ & $\begin{array}{l}\text { Recovered } \\
\quad(1 \mathrm{mo})\end{array}$ \\
\hline $\begin{array}{l}\text { Jiang and Chen, } 1987 \\
\text { [146] }\end{array}$ & 1 & $77 / \mathrm{F}$ & Stiff neck & GB20 & $\begin{array}{c}\text { The } \\
\text { cerebellopontine } \\
\text { and subarachnoid } \\
\text { hemorrhage }\end{array}$ & After treatment & Dead \\
\hline Zhou, 1988 [147] & 1 & $15 / \mathrm{M}$ & Cold & GB20 & $\begin{array}{l}\text { Subarachnoid } \\
\text { hemorrhage }\end{array}$ & $\begin{array}{l}\text { During the } \\
\text { treatment }\end{array}$ & Dead \\
\hline Mi et al., 1989 [148] & 1 & $28 / \mathrm{F}$ & Neurosis & DU15 & $\begin{array}{l}\text { Subarachnoid } \\
\text { hemorrhage }\end{array}$ & $2 \mathrm{~d}$ & $\begin{array}{l}\text { Recovered } \\
\quad(27 \mathrm{~d})\end{array}$ \\
\hline Wu and Xu, 1990 [149] & 1 & $57 / \mathrm{M}$ & Stroke & $\begin{array}{l}\text { Ashi points near } \\
\text { C3 }\end{array}$ & $\begin{array}{l}\text { Subarachnoid } \\
\text { hemorrhage }\end{array}$ & $1 \mathrm{~h}$ & Dead $(10 \mathrm{~d})$ \\
\hline
\end{tabular}


TABLE 2: Continued.

\begin{tabular}{|c|c|c|c|c|c|c|c|}
\hline Author/Year (reference) & Cases & Age/Sex & Disease treated & Punctured site & Complication & $\begin{array}{c}\text { Onset after } \\
\text { acupuncture }\end{array}$ & Follow-up \\
\hline Liu, 1992 [150] & 1 & $28 / \mathrm{M}$ & Insomnia & GB20 & $\begin{array}{l}\text { Acute subdural } \\
\text { hematoma }\end{array}$ & 3 hours & $\begin{array}{l}\text { Recovered } \\
\text { after surgery }\end{array}$ \\
\hline $\mathrm{Mi}, 1993$ [12] & 1 & $73 / \mathrm{M}$ & $\begin{array}{c}\text { Cerebral } \\
\text { hemorrhage }\end{array}$ & $\begin{array}{c}\text { LI11, LI4, GB30, } \\
\text { ST36, GB39, } \\
\text { GB14, ST2 }\end{array}$ & $\begin{array}{c}\text { Cerebral } \\
\text { hemorrhage } \\
\text { reformulation }\end{array}$ & $10 \mathrm{~min}$ & Dead \\
\hline Jiang et al., 1996 [151] & 2 & $\begin{array}{l}45 / \mathrm{M} \\
54 / \mathrm{M}\end{array}$ & $\begin{array}{l}\text { Cervical } \\
\text { spondylopathy }\end{array}$ & $\begin{array}{l}\text { GB20 } \\
\text { Neck }\end{array}$ & $\begin{array}{l}\text { Subarachnoid } \\
\text { hemorrhage }\end{array}$ & Immediately & $\begin{array}{l}\text { Recovered } \\
\quad(30 \mathrm{~d}) \\
\text { Recovered } \\
(1 \mathrm{mo})\end{array}$ \\
\hline Liu et al., 1996 [152] & 1 & $35 / \mathrm{M}$ & $\begin{array}{l}\text { Ankylosing } \\
\text { Spondylitis }\end{array}$ & DU16 & $\begin{array}{l}\text { Medulla oblongata } \\
\text { hemorrhage }\end{array}$ & 5 hours & Dead \\
\hline Bian et al., 1997 [153] & 1 & $29 / \mathrm{F}$ & $\begin{array}{c}\text { Headache caused } \\
\text { by pesticide } \\
\text { poisoning }\end{array}$ & GB20 & $\begin{array}{l}\text { Subarachnoid } \\
\text { hemorrhage }\end{array}$ & $3 \mathrm{~min}$ & $\begin{array}{l}\text { Recovered } \\
\quad(1 \mathrm{mo})\end{array}$ \\
\hline Wang, 1999 [10] & 1 & $54 / \mathrm{M}$ & Low back pain & BL37 & $\begin{array}{c}\text { Acute } \\
\text { subarachnoid } \\
\text { hemorrhage }\end{array}$ & Immediately & Recovered \\
\hline Wang, 1999 [154] & 1 & $39 / \mathrm{F}$ & Neurosis & GB20 & $\begin{array}{l}\text { Cervical cord } \\
\text { injury }\end{array}$ & Immediately & Dead \\
\hline Song and $\mathrm{Wu}, 2001$ [9] & 1 & $58 / \mathrm{M}$ & $\begin{array}{l}\text { Hypertensive } \\
\text { cerebral } \\
\text { hemorrhage }\end{array}$ & KI01 & $\begin{array}{c}\text { Cerebral } \\
\text { hemorrhage } \\
\text { reformulation }\end{array}$ & Immediately & Dead \\
\hline Li et al., 2003 [155] & 1 & $55 / \mathrm{M}$ & $\begin{array}{l}\text { Neck and back } \\
\text { pain }\end{array}$ & Neck and back & $\begin{array}{c}\text { Cervical spinal } \\
\text { epidural hematoma }\end{array}$ & Immediately & Recovered \\
\hline $\begin{array}{l}\text { Niu and Zhang, } 2006 \\
{[156]}\end{array}$ & 1 & $42 / \mathrm{M}$ & Headache & Neck & $\begin{array}{l}\text { Cisterna magna } \\
\text { hemorrhage }\end{array}$ & Not specified & $\begin{array}{l}\text { Recovered } \\
\text { after surgery }\end{array}$ \\
\hline Li et al., 2008 [157] & 1 & $36 / \mathrm{F}$ & Migraine & DU16 & $\begin{array}{l}\text { Subarachnoid } \\
\text { hemorrhage }\end{array}$ & At night & $\begin{array}{l}\text { Recovered } \\
(3 \mathrm{wk})\end{array}$ \\
\hline Chen, 2009 [11] & 1 & $43 / \mathrm{F}$ & Lumbago pain & Waist & $\begin{array}{l}\text { Subarachnoid } \\
\text { hemorrhage }\end{array}$ & Immediately & $\begin{array}{l}\text { Recovered } \\
\quad(1 \mathrm{mo})\end{array}$ \\
\hline Li et al., 2011 [13] & 1 & $45 / \mathrm{F}$ & Toothache & $\begin{array}{l}\text { RN16, RN10, } \\
\text { RN9, RN4 }\end{array}$ & $\begin{array}{c}\text { Leukemia acute } \\
\text { intracerebral } \\
\text { hemorrhage }\end{array}$ & Immediately & Dead $(2 d)$ \\
\hline
\end{tabular}

TABLE 3: Peripheral nerve injury.

\begin{tabular}{|c|c|c|c|c|c|c|c|}
\hline Author/year (reference) & Cases & Age/sex & Disease treated & Punctured site & Complication & Practitioner & Follow-up \\
\hline Tang and Fang, 1986 [15] & 1 & $\begin{array}{c}\text { Not } \\
\text { specified }\end{array}$ & Facial paralysis & Not specified & $\begin{array}{c}\text { Aggravated } \\
\text { facial paralysis }\end{array}$ & Not specified & Recovered \\
\hline Nie and Zhou, 1990 [16] & 1 & $26 / \mathrm{M}$ & Left eye pain & SJ17 & Cardiac arrest & Acupuncturist & $\begin{array}{l}\text { Recovered } \\
\quad(1 \mathrm{~min})\end{array}$ \\
\hline Yan, 1994 [17] & 1 & $57 / \mathrm{M}$ & $\begin{array}{l}\text { Cervical } \\
\text { dislocation }\end{array}$ & Bitong point & $\begin{array}{l}\text { Phrenic nerve } \\
\text { injury }\end{array}$ & Acupuncturist & $\begin{array}{l}\text { Recovered } \\
\quad(1 \mathrm{wk})\end{array}$ \\
\hline Yang and Wang, 1996 [18] & 1 & $62 / \mathrm{M}$ & $\begin{array}{c}\text { Lateral rectus } \\
\text { paralysis }\end{array}$ & Ex-HN07 & Optic atrophy & Acupuncturist & Blind \\
\hline Xu and Liu, 1997 [19] & 1 & $48 / \mathrm{M}$ & $\begin{array}{l}\text { Tinnitus and } \\
\text { hearing loss }\end{array}$ & $\begin{array}{l}\text { ST1, ST2, BL2, } \\
\text { LI20, SJ21, SI19 }\end{array}$ & $\begin{array}{l}\text { Oculomotorius } \\
\text { injury }\end{array}$ & Not specified & $\begin{array}{l}\text { Recovered } \\
\quad(17 \mathrm{~d})\end{array}$ \\
\hline Huang and Wei, 1997 [20] & 1 & $53 / \mathrm{F}$ & $\begin{array}{l}\text { Trigeminal } \\
\text { nerve pain }\end{array}$ & $\begin{array}{l}\text { Around the } \\
\text { mandible }\end{array}$ & $\begin{array}{l}\text { Trigeminal } \\
\text { nerve injury }\end{array}$ & $\begin{array}{l}\text { Acupuncturist } \\
\text { in the } \\
\text { stomatological } \\
\text { hospital }\end{array}$ & $\begin{array}{l}\text { Improved } \\
(3 \mathrm{mo})\end{array}$ \\
\hline Wang, 1999 [10] & 1 & $54 / \mathrm{F}$ & Lumbago & GB30 & $\begin{array}{c}\text { Sciatic nerve } \\
\text { injury }\end{array}$ & $\begin{array}{c}\text { Private } \\
\text { practitioner }\end{array}$ & Unrecovered \\
\hline Ruan et al., 2009 [21] & 1 & $67 / \mathrm{F}$ & Lumbago & GB34 & $\begin{array}{l}\text { Peroneus nerve } \\
\text { injury }\end{array}$ & Not specified & $\begin{array}{l}\text { Recovered } \\
(3 \mathrm{wk})\end{array}$ \\
\hline
\end{tabular}


TABLE 4: Organ injury associated with acupuncture.

\begin{tabular}{|c|c|c|c|c|c|c|}
\hline Author/year (reference) & Cases & Age/sex & Disease treated & Punctured site & Complication & Follow-up \\
\hline Liu, $1981[14]$ & 1 & $19 / \mathrm{F}$ & Schizophrenia & RN15 & $\begin{array}{l}\text { Cardiac } \\
\text { tamponade }\end{array}$ & Dead \\
\hline Zhu, 1990 [22] & 1 & $64 / \mathrm{M}$ & $\begin{array}{l}\text { Chest stiffness and } \\
\text { rib pain }\end{array}$ & LR14 & Aortoclasia & Dead \\
\hline Shi, 1993 [23] & 1 & $42 / \mathrm{F}$ & Witchcraft & $\begin{array}{l}\text { Limbs, chest, } \\
\text { abdomen, back }\end{array}$ & Aortoclasia & Dead \\
\hline Xie and Lin, 2003 [24] & 1 & $35 / \mathrm{F}$ & $\begin{array}{c}\text { Intercostal } \\
\text { neuralgia }\end{array}$ & Right breast & Cardiac trauma & Dead \\
\hline Luo et al., 2006 [25] & 1 & $40 / \mathrm{M}$ & $\begin{array}{l}\text { Chronic } \\
\text { esophagitis }\end{array}$ & RN15 & $\begin{array}{c}\text { Cardiac } \\
\text { tamponade }\end{array}$ & Recovered \\
\hline Zhang and Dong, 2006 [26] & 1 & $37 / \mathrm{M}$ & $\begin{array}{l}\text { Chest tightness, } \\
\text { asthma }\end{array}$ & Chest and back & $\begin{array}{c}\text { Cardiac } \\
\text { tamponade }\end{array}$ & Dead \\
\hline Zhu et al., 2008 [27] & 1 & $44 / \mathrm{F}$ & Diabetes & Chest & $\begin{array}{c}\text { Cardiac } \\
\text { tamponade }\end{array}$ & Dead \\
\hline Yang, 1991 [28] & 1 & $21 / \mathrm{M}$ & Bad cold & BL13 & Chylothorax & Recovered (2wk) \\
\hline \multirow{2}{*}{ Zheng and Zhao, 1983 [30] } & 1 & $31 / \mathrm{F}$ & Stomachache & ST25, LR14, RN12 & \multirow{2}{*}{$\begin{array}{c}\text { Gallbladder } \\
\text { perforation and } \\
\text { biliary peritonitis }\end{array}$} & \multirow[b]{2}{*}{$\begin{array}{l}\text { Recovered after } \\
\text { surgery }\end{array}$} \\
\hline & 1 & $59 / \mathrm{M}$ & $\begin{array}{c}\text { Cervical } \\
\text { spondylopathy }\end{array}$ & RN12, RN13, ST25 & & \\
\hline Deng, 1985 [31] & 1 & $45 / \mathrm{F}$ & $\begin{array}{l}\text { Acute attack of } \\
\text { chronic } \\
\text { cholecystitis }\end{array}$ & ST21 & $\begin{array}{l}\text { Gallbladder } \\
\text { perforation }\end{array}$ & Not specified \\
\hline Bai, $1991[32]$ & 1 & $32 / \mathrm{F}$ & Paralytic ileus & Upper abdomen & $\begin{array}{c}\text { Gallbladder } \\
\text { perforation and } \\
\text { biliary peritonitis }\end{array}$ & $\begin{array}{l}\text { Recovered after } \\
\text { surgery }\end{array}$ \\
\hline Duan and Wang, 1984 [29] & 1 & $42 / \mathrm{M}$ & Intestinal spasm & RN8, RN12, ST25 & $\begin{array}{l}\text { Intestinal } \\
\text { perforation and } \\
\text { suppurative } \\
\text { peritonitis }\end{array}$ & $\begin{array}{l}\text { Recovered after } \\
\text { surgery (14 d) }\end{array}$ \\
\hline \multirow{4}{*}{ Zhang, 1997 [33] } & \multirow{4}{*}{4} & $51 / \mathrm{M}$ & $\begin{array}{c}\text { Paroxysmal } \\
\text { abdominal pain }\end{array}$ & \multirow{4}{*}{ Abdomen } & \multirow{4}{*}{$\begin{array}{l}\text { Localized } \\
\text { peritonitis }\end{array}$} & \multirow{4}{*}{$\begin{array}{l}\text { Recovered } \\
\text { after surgery }\end{array}$} \\
\hline & & $47 / \mathrm{F}$ & $\begin{array}{c}\text { Right lower } \\
\text { abdominal pain }\end{array}$ & & & \\
\hline & & $53 / \mathrm{F}$ & $\begin{array}{l}\text { Abdominal pain, } \\
\text { diarrhea }\end{array}$ & & & \\
\hline & & $37 / \mathrm{F}$ & Periumbilical pain & & & \\
\hline Xiao, 1985 [34] & 1 & $20 / M$ & $\begin{array}{l}\text { Abdominal } \\
\text { discomfort }\end{array}$ & RN12 & Gastric perforation & $\begin{array}{l}\text { Recovered after } \\
\text { surgery }(11 \mathrm{~d})\end{array}$ \\
\hline Huang, 1999 [35] & 1 & $54 / \mathrm{M}$ & Gastric ulcer & ST36 & $\begin{array}{l}\text { Gastric ulcer with } \\
\text { perforation }\end{array}$ & $\begin{array}{l}\text { Recovered after } \\
\text { surgery }(10 \mathrm{~d})\end{array}$ \\
\hline Tang et al., 2006 [36] & 1 & $61 / \mathrm{M}$ & RA & $\begin{array}{l}\text { ST34, ST40, SP6, } \\
\text { SP10, GB33, GB34 }\end{array}$ & $\begin{array}{c}\text { Gastric stress ulcer } \\
\text { and hemorrhagic } \\
\text { shock }\end{array}$ & Recovered (2 mo) \\
\hline Liu et al., 1992 [37] & 1 & $2 / \mathrm{M}$ & Diarrhea & RN12, ST25, DU1 & $\begin{array}{l}\text { Complete } \\
\text { intestinal } \\
\text { obstruction }\end{array}$ & $\begin{array}{l}\text { Recovered after } \\
\text { surgery }\end{array}$ \\
\hline Zhang et al., 1992 [38] & 1 & $45 / \mathrm{F}$ & $\begin{array}{l}\text { Chronic bronchitis, } \\
\text { coronary disease }\end{array}$ & GB21, BL13, BL23 & $\begin{array}{l}\text { Multiple organ } \\
\text { injury }\end{array}$ & Dead \\
\hline
\end{tabular}

One patient suffered from retinal detachment, and eyesight was corrected to 0.2 after treatment [43]. One patient lost eyesight because of traumatic cataract [18]. One patient experienced femoral neck fracture after strong stimulation to myospasm [45]. One patient suffered from subluxation in right wrist joint due to excessive EA intensity [48].
3.7. Syncope (Table 6). A total of 18 cases of acupunctureassociated syncope were reported (Table 6). Syncope occurred during the treatment in 9 cases, several minutes after the treatment in 5 cases, and several hours later in 4 patients. Two patients fainted after taking liquors $[51,52]$. The positions were sitting $(n=5)$, lying (5), and not specified (8). 
TABLE 5: Other tissue injury.

\begin{tabular}{|c|c|c|c|c|c|c|c|}
\hline Author/year (reference) & Cases & Age/sex & Disease treated & $\begin{array}{c}\text { Punctured } \\
\text { site }\end{array}$ & Complication & Practitioner & Follow-up \\
\hline Chen, 1980 [39] & 1 & $33 / \mathrm{F}$ & Sore throat & Neck & $\begin{array}{l}\text { Cervical common } \\
\text { carotid aneurysm }\end{array}$ & Not specified & $\begin{array}{l}\text { Recovered } \\
\text { after surgery } \\
(3 \mathrm{mo})\end{array}$ \\
\hline Wang, $1987[40]$ & 1 & $42 / \mathrm{F}$ & $\begin{array}{l}\text { Thyroid cancer } \\
\text { (anaesthesia for } \\
\text { thyroidectomy) }\end{array}$ & SJ17 & $\begin{array}{l}\text { Sinus caroticus } \\
\text { syndrome, shock }\end{array}$ & Not specified & Recovered \\
\hline Zhang et al., 1992 [38] & 1 & $39 / \mathrm{M}$ & Asthma & BL13 & Pleural shock & Not specified & $\begin{array}{l}\text { Recovered } \\
\qquad(2 \mathrm{~d})\end{array}$ \\
\hline Zhu, 1986 [41] & 1 & $56 / \mathrm{M}$ & Chest distress & RN22 & Asphyxia & Acupuncturist & Dead \\
\hline Gao, 1989 [42] & 1 & $73 / \mathrm{M}$ & Cough & LI18 & Dyspnea & Not specified & $\begin{array}{l}\text { Recovered } \\
\quad(3 \mathrm{~d})\end{array}$ \\
\hline Liu et al., 1988 [43] & 1 & $53 / \mathrm{M}$ & Blepharospasm & $\begin{array}{l}\text { The lateral } \\
\text { upper eyelid }\end{array}$ & $\begin{array}{c}\text { Retinal } \\
\text { detachment }\end{array}$ & Health worker & $\begin{array}{c}\text { Recovered } \\
\text { after surgery }\end{array}$ \\
\hline Yang and Wang, 1996 [18] & 1 & $63 / F$ & Left eye ptosis & Periocular & Traumatic cataract & Acupuncturist & Blind \\
\hline \multirow[t]{2}{*}{ Wang, $1982[44]$} & \multirow[t]{2}{*}{2} & $20 / \mathrm{M}$ & $\begin{array}{l}\text { Traumatic } \\
\text { mydriasis }\end{array}$ & \multirow[t]{2}{*}{ EX-HN5 } & $\begin{array}{c}\text { The orbicularis } \\
\text { oculi muscle } \\
\text { tremor }\end{array}$ & \multirow[t]{2}{*}{ Not specified } & $\begin{array}{l}\text { Recovered } \\
\quad(3 \mathrm{~d})\end{array}$ \\
\hline & & $35 / F$ & Right migraine & & $\begin{array}{l}\text { Speech and } \\
\text { swallowing } \\
\text { difficulties }\end{array}$ & & $\begin{array}{l}\text { Recovered } \\
\quad(2 \mathrm{~h})\end{array}$ \\
\hline Li and Zhou, 1980 [45] & 1 & $40 / \mathrm{M}$ & Schizophrenia & EX-HN5 & $\begin{array}{l}\text { Femoral neck } \\
\text { fracture }\end{array}$ & Acupuncturist & Not specified \\
\hline Liang and Song, 1984 [46] & 1 & $23 / \mathrm{M}$ & Cramp & BL57 & $\begin{array}{l}\text { Flexor hallucis } \\
\text { muscle and } \\
\text { digitorum longus } \\
\text { muscle scar } \\
\text { contracture }\end{array}$ & Health worker & $\begin{array}{l}\text { Recovered } \\
\text { after surgery }\end{array}$ \\
\hline Chen, 1992 [47] & 1 & $17 / \mathrm{F}$ & Shortsightedness & $\begin{array}{c}\text { GB20, } \\
\text { EX-HN17 }\end{array}$ & Nape spasm & Acupuncturist & $\begin{array}{l}\text { Recovered } \\
\quad(6 \mathrm{~d})\end{array}$ \\
\hline Wang, 1994 [48] & 1 & $65 / F$ & Stroke & LI4, SJ5, LI15 & $\begin{array}{l}\text { Subluxation of } \\
\text { wrist }\end{array}$ & Intern & $\begin{array}{l}\text { Recovered } \\
\quad(1 \mathrm{wk})\end{array}$ \\
\hline \multirow{2}{*}{ Shi and Chen, 1994 [49] } & 1 & $52 / \mathrm{M}$ & Facioplegia & LI11 & Elbow pain & \multirow{2}{*}{ Acupuncturist } & $\begin{array}{l}\text { Recovered } \\
(2 \mathrm{wk})\end{array}$ \\
\hline & 1 & $61 / F$ & $\begin{array}{l}\text { Scapulohumeral } \\
\text { periarthritis }\end{array}$ & Not specified & Shoulder pain & & $\begin{array}{l}\text { Recovered } \\
\quad(20 \mathrm{~d})\end{array}$ \\
\hline Wang, 1999 [10] & 1 & $53 / \mathrm{M}$ & Cough, epistaxis & LU6 & Amyotrophy & $\begin{array}{c}\text { Private } \\
\text { practitioner }\end{array}$ & Unrecovered \\
\hline \multirow{2}{*}{ Luo and Huang, 2006 [50] } & \multirow[t]{2}{*}{2} & $42 / \mathrm{M}$ & Amyotrophic & Upper limb & \multirow{2}{*}{ Fasciculation } & \multirow{2}{*}{ Not specified } & Improved \\
\hline & & $63 / \mathrm{M}$ & & Upper limb & & & Improved \\
\hline
\end{tabular}

Most patients recovered with rest and drinking sugar water, while 2 patients recovered after injection of metoclopramide via ST36 [53]. Two cases suffered from sudden cardiac arrest and were cured after first aid $[54,55]$. One patient recovered after massaging an ear acupoint [56].

3.8. Infection (Table 7). A total of 17 cases were infections associated with acupuncture (Table 7). Among them, 10 recovered, 3 died, 2 improved after 3 days, 1 was disabled, and 1 was not stated. The infection was caused by tetanus bacillus $(n=6)$ [10,57-61], hydatid (1) [62], Escherichia coli (1) [63], and Mycobacterium tuberculosis (3) [64]. One patient was infected after deep 3-degree burning [65] while others were not stated.
In one patient, the right epigastric mass due to acupunctured liver hydatid caused extensive metastasis in hydatid abdominal cavity; the patient recovered after operation [62]. One diabetic patient without controlling blood glucose suffered from diabetic feet because of infection and recovered after hypoglycemic and anti-infection treatments.

3.9. Hemorrhage (Table 8). Among 10 cases of acupunctureinduced local hemorrhage (Table 8 ), 8 patients recovered, 1 improved, but 1 died. The positions of hemorrhage included eyes $(n=2)[6,66]$, extraperitoneal (1) [67], thyroid (1) [68], hypoglossus $(2)[69,70]$, suffocated death from hematoma compressed trachea (1) [71], hand (1) [72], 1 case of buttock 
TABLE 6: Syncope associated with acupuncture.

\begin{tabular}{|c|c|c|c|c|c|c|c|c|c|}
\hline $\begin{array}{l}\text { Author/year } \\
\text { (reference) }\end{array}$ & Cases & Age/sex & Disease treated & $\begin{array}{l}\text { Punctured } \\
\text { sites }\end{array}$ & Position & Start time & Causes & Practitioner & Follow-up \\
\hline $\begin{array}{l}\text { Yang, } 1986 \\
{[6]}\end{array}$ & 1 & $32 / \mathrm{M}$ & Facioplegia & $\begin{array}{l}\text { GB14, ST6, } \\
\text { ST4, SJ17, } \\
\text { LI20, LI4 }\end{array}$ & Sitting & $\begin{array}{c}5 \text { min after } \\
\text { inserting } \\
\text { needle }\end{array}$ & Limosis & Acupuncturist & Recovered \\
\hline $\begin{array}{l}\text { Shao, } 1989 \\
{[51]}\end{array}$ & 1 & $53 / \mathrm{M}$ & $\begin{array}{c}\text { Myotenositis of } \\
\text { long head of } \\
\text { biceps brachii }\end{array}$ & $\begin{array}{l}\text { LI15, LI11, } \\
\text { ashi }\end{array}$ & Not specified & $\begin{array}{l}\text { After } \\
\text { inserting } \\
\text { needle }\end{array}$ & $\begin{array}{c}\text { After } \\
\text { drinking }\end{array}$ & Acupuncturist & $\begin{array}{l}\text { Recovered } \\
\quad(20 \mathrm{~m})\end{array}$ \\
\hline $\begin{array}{l}\text { Shi and } \\
\text { Chen, } 1994 \\
{[49]}\end{array}$ & 1 & $57 / F$ & Stomachache & $\begin{array}{l}\text { LI3, ST36, } \\
\text { PC6 }\end{array}$ & Not specified & $\begin{array}{c}\text { Finish } \\
\text { needling }\end{array}$ & Limosis & Acupuncturist & $\begin{array}{c}\text { Recovered } \\
(30 \mathrm{~min})\end{array}$ \\
\hline \multirow{2}{*}{$\begin{array}{l}\text { Guo, } 1995 \\
{[158]}\end{array}$} & \multirow[b]{2}{*}{2} & $65 / F$ & $\begin{array}{c}\text { Scapulohumeral } \\
\text { periarthritis }\end{array}$ & $\begin{array}{l}\text { Ex-UE01, } \\
\text { GB21, LI14, } \\
\text { LI11, SJ5, LI4 }\end{array}$ & \multirow[b]{2}{*}{ Clinostatism } & $\begin{array}{c}\text { Finish } \\
\text { needling }\end{array}$ & Weakness & Acupuncturist & Recovered \\
\hline & & $24 / \mathrm{F}$ & Waist sprain & $\begin{array}{l}\text { BL40, ashi } \\
\text { point and } \\
\text { acupoint of } \\
\text { bladder } \\
\text { meridian }\end{array}$ & & $\begin{array}{c}\text { Finish } \\
\text { needling }\end{array}$ & Nervous & Acupuncturist & $\begin{array}{l}\text { Recovered } \\
(10 \mathrm{~min})\end{array}$ \\
\hline \multirow[t]{2}{*}{$\begin{array}{l}\text { Wu et al., } \\
2001[56]\end{array}$} & \multirow[t]{2}{*}{1} & $24 / \mathrm{F}$ & Insomnia & $\begin{array}{c}\text { PC6, ST36, } \\
\text { HT7 }\end{array}$ & Not specified & At night & $\begin{array}{c}\text { Not } \\
\text { specified }\end{array}$ & Acupuncturist & $\begin{array}{l}\text { Recovered } \\
\quad(2 \mathrm{~d})\end{array}$ \\
\hline & & $45 / \mathrm{F}$ & Lumbago pain & $\begin{array}{l}\text { BL23, BL40, } \\
\text { DU3, GB30, } \\
\text { KI7 }\end{array}$ & Not specified & $\begin{array}{c}\text { Finish } \\
\text { needling }\end{array}$ & $\begin{array}{c}\text { After } \\
\text { drinking }\end{array}$ & Acupuncturist & \\
\hline \multirow[t]{2}{*}{ Liu, 2001 [52] } & \multirow[t]{2}{*}{3} & $34 / \mathrm{F}$ & Shoulder pain & $\begin{array}{l}\text { LI11, LI14, } \\
\text { LI15, SJ5, SJ14 }\end{array}$ & Sitting & $\begin{array}{c}\text { After } \\
\text { inserting } \\
\text { needle }\end{array}$ & Tiredness & Acupuncturist & Recoverec \\
\hline & & $56 / \mathrm{F}$ & $\begin{array}{l}\text { Right thumb } \\
\text { pain }\end{array}$ & $\begin{array}{l}\text { LI4, LI5, LI11, } \\
\text { ashi point }\end{array}$ & Not specified & $\begin{array}{c}\text { On the way } \\
\text { home }\end{array}$ & Limosis & Acupuncturist & \\
\hline \multirow[t]{2}{*}{$\mathrm{Ma}, 2005[54]$} & \multirow[t]{2}{*}{1} & $28 / \mathrm{M}$ & Prosopalgia & $\begin{array}{c}\text { EX-HN5, } \\
\text { LI4, ST6, SJ5 }\end{array}$ & Sitting & $\begin{array}{c}5 \text { min after } \\
\text { inserting } \\
\text { needle }\end{array}$ & $\begin{array}{c}\text { Not } \\
\text { specified }\end{array}$ & Acupuncturist & $\begin{array}{l}\text { Recovered } \\
\quad(30 \mathrm{~s})\end{array}$ \\
\hline & & $72 / \mathrm{M}$ & Stroke & $\begin{array}{l}\text { LI11, SJ5, } \\
\text { ST36, SP6, } \\
\text { EX-UE17, } \\
\text { EX-LE11 }\end{array}$ & Clinostatism & $\begin{array}{l}10 \text { min after } \\
\text { inserting } \\
\text { needle }\end{array}$ & Nervous & Acupuncturist & $\begin{array}{l}\text { Recovered } \\
(2 \mathrm{~min})\end{array}$ \\
\hline \multirow[t]{2}{*}{$\begin{array}{l}\text { Long et al., } \\
2006[55]\end{array}$} & \multirow[t]{2}{*}{3} & $41 / \mathrm{F}$ & $\begin{array}{c}\text { Cervical } \\
\text { spondylopathy }\end{array}$ & BL10, GB20 & Sitting & $\begin{array}{c}1 \text { min after } \\
\text { inserting } \\
\text { needle }\end{array}$ & $\begin{array}{c}\text { Not } \\
\text { specified }\end{array}$ & Acupuncturist & $\begin{array}{c}\text { Recovered } \\
(30 \mathrm{~min})\end{array}$ \\
\hline & & $42 / \mathrm{F}$ & Lumbago pain & ST36 & Clinostatism & $\begin{array}{l}\text { After } \\
\text { inserting } \\
\text { needle }\end{array}$ & $\begin{array}{l}\text { Heavy } \\
\text { stimulus }\end{array}$ & Acupuncturist & $\begin{array}{l}\text { Recovered } \\
(2 \mathrm{~min})\end{array}$ \\
\hline $\begin{array}{l}\text { Liu, } 2007 \\
{[159]}\end{array}$ & 1 & $42 / \mathrm{F}$ & $\begin{array}{c}\text { Scapulohumeral } \\
\text { periarthritis }\end{array}$ & $\begin{array}{l}\text { Ex-UE01, } \\
\text { LI14, Ashi } \\
\text { point, SJ3 }\end{array}$ & Sitting & $\begin{array}{l}10 \text { min after } \\
\text { inserting } \\
\text { needle }\end{array}$ & $\begin{array}{c}\text { Not } \\
\text { specified }\end{array}$ & Acupuncturist & $\begin{array}{c}\text { Recovered } \\
(30 \mathrm{~min})\end{array}$ \\
\hline $\begin{array}{l}\text { Chen, } 2009 \\
{[11]}\end{array}$ & 1 & $42 / \mathrm{M}$ & $\begin{array}{l}\text { Acute lumbar } \\
\text { sprain }\end{array}$ & EX-B5, BL40 & Not specified & $\begin{array}{c}\text { Finish } \\
\text { needling }\end{array}$ & $\begin{array}{c}\text { Not } \\
\text { specified }\end{array}$ & Acupuncturist & $\begin{array}{l}\text { Recovered } \\
(10 \mathrm{~min})\end{array}$ \\
\hline $\begin{array}{l}\text { Liao and } \\
\text { Guo, } 2009 \\
{[160]}\end{array}$ & 1 & $57 / \mathrm{F}$ & Gouty arthritis & GB20 & Clinostatism & $\begin{array}{l}\text { After } \\
\text { inserting } \\
\text { needle }\end{array}$ & Limosis & Acupuncturist & $\begin{array}{l}\text { Recovered } \\
(10 \mathrm{~min})\end{array}$ \\
\hline \multirow{2}{*}{$\begin{array}{l}\text { Li et al., } 2009 \\
\text { [53] }\end{array}$} & \multirow[b]{2}{*}{2} & $48 / \mathrm{M}$ & $\begin{array}{l}\text { Cervical } \\
\text { spondylopathy } \\
\text { radiculaire }\end{array}$ & $\begin{array}{l}\text { GB21, LI11, } \\
\text { SJ5, Ex-B5 }\end{array}$ & & $\begin{array}{l}6 \mathrm{~h} \text { after } \\
\text { treatment }\end{array}$ & $\begin{array}{c}\text { Not } \\
\text { specified }\end{array}$ & Acupuncturist & $\begin{array}{c}\text { Recovered } \\
\text { after stop } \\
\text { treatment }\end{array}$ \\
\hline & & $68 / \mathrm{F}$ & Facial paralysis & $\begin{array}{l}\text { GB14, ST2, } \\
\text { ST4, ST6, } \\
\text { SJ17, LI4, } \\
\text { Ex-HN16 }\end{array}$ & Not specified & $\begin{array}{l}12 \mathrm{~h} \text { after } \\
\text { treatment }\end{array}$ & $\begin{array}{c}\text { Not } \\
\text { specified }\end{array}$ & Acupuncturist & $\begin{array}{l}\text { Recovered } \\
\text { after stop } \\
\text { treatment }\end{array}$ \\
\hline
\end{tabular}


TABLE 7: Infection associated with acupuncture.

\begin{tabular}{|c|c|c|c|c|c|c|c|}
\hline Author/year (reference) & Cases & Age/sex & Disease treated & $\begin{array}{c}\text { Punctured } \\
\text { site }\end{array}$ & Diagnosis & Practitioner & Follow-up \\
\hline Zhang, 1980 [161] & 1 & $5 / \mathrm{F}$ & Heat, cough & Ex-UE19 & Infection & Not specified & $\begin{array}{l}\text { Middle finger } \\
\text { disability }\end{array}$ \\
\hline Xie and Zong, 1983 [62] & 1 & $38 / \mathrm{F}$ & $\begin{array}{c}\text { Right upper } \\
\text { abdominal mass } \\
\text { and discomfort }\end{array}$ & Ashi points & $\begin{array}{c}\text { Abdominal } \\
\text { metastatic hepatic } \\
\text { hydatid }\end{array}$ & Local hospital & $\begin{array}{l}\text { Recovered } \\
\text { after surgery }\end{array}$ \\
\hline Gao and Qi, 1989 [65] & 1 & $54 / \mathrm{M}$ & Right leg pain & Local points & $\begin{array}{l}\text { Third-degree burns } \\
\text { with infection }\end{array}$ & Clinic & Not specified \\
\hline $\mathrm{Xu}, 1990$ [162] & 1 & $19 / \mathrm{M}$ & Migraine & $\begin{array}{c}\text { EX-HN5, } \\
\text { GB14, DU20 }\end{array}$ & $\begin{array}{c}\text { Head abscess, } \\
\text { intracranial } \\
\text { infection }\end{array}$ & $\begin{array}{l}\text { Health worker } \\
\text { in the army }\end{array}$ & Recovered \\
\hline Xia, 1993 [129] & 1 & $37 / \mathrm{F}$ & Facioplegia & ST5 & $\begin{array}{l}\text { Right cheek } \\
\text { hematoma with } \\
\text { infection }\end{array}$ & Not specified & $\begin{array}{l}\text { Recovered } \\
\quad(1 \mathrm{wk})\end{array}$ \\
\hline Chen and Gao, 1995 [63] & 1 & $30 / \mathrm{M}$ & Insomnia & Head & Encephalopyosis & Not specified & $\begin{array}{c}\text { Recovered } \\
\text { after surgery }\end{array}$ \\
\hline Zhou, 1999 [64] & 3 & $\begin{array}{l}\text { From } 22 \text { to } \\
\text { 28/M (1); F } \\
\text { (2) }\end{array}$ & $\begin{array}{c}\text { Lumbar muscle } \\
\text { strain }\end{array}$ & Loin & $\begin{array}{l}\text { Tuberculous } \\
\text { abscess on the } \\
\text { body surface }\end{array}$ & Not specified & $\begin{array}{l}\text { Recovered } \\
(3-6 \mathrm{mo})\end{array}$ \\
\hline Song and $\mathrm{Wu}, 2001$ [9] & 1 & $38 / \mathrm{M}$ & $\begin{array}{l}\text { Rheumatic } \\
\text { arthritis }\end{array}$ & $\begin{array}{l}\text { EX-LE4, } \\
\text { EX-LE5, } \\
\text { GB34, SP9 }\end{array}$ & Pyogenic arthritis & $\begin{array}{l}\text { Individual clinic } \\
\text { in the country }\end{array}$ & $\begin{array}{l}\text { Recovered } \\
(2 \mathrm{wk})\end{array}$ \\
\hline Ding et al., 2008 [112] & 1 & $60 / \mathrm{F}$ & $\begin{array}{c}\text { Scapulohumeral } \\
\text { periarthritis }\end{array}$ & ST38 & Diabetes foot & Not specified & $\begin{array}{l}\text { Remission } \\
\text { after } 3 \text { days }\end{array}$ \\
\hline Yang et al., 1990 [57] & 1 & $2 / \mathrm{F}$ & $\begin{array}{l}\text { Malnutritional } \\
\text { stagnation }\end{array}$ & Ex-UE19 & Tetanus & Illegal treatment & Dead \\
\hline Liu, $1991[58]$ & 1 & $52 / \mathrm{F}$ & Headache & $\begin{array}{c}\text { Ex-HN05, } \\
\text { DU20, GB20, } \\
\text { LI4 }\end{array}$ & Tetanus & $\begin{array}{c}\text { Village } \\
\text { acupuncturist }\end{array}$ & $\begin{array}{l}\text { Recovered } \\
(2 \mathrm{wk})\end{array}$ \\
\hline Liu, 1992 [59] & 1 & $52 / \mathrm{F}$ & Leg pain & Not specified & Tetanus & $\begin{array}{c}\text { Private } \\
\text { practitioner }\end{array}$ & $\begin{array}{l}\text { Recovered } \\
\quad(1 \mathrm{mo})\end{array}$ \\
\hline Sun and $\mathrm{Hu}, 1997$ [60] & 1 & $23 / \mathrm{M}$ & Facioplegia & Not specified & Tetanus & Health-center & $\begin{array}{l}\text { Remission } \\
\text { after } 3 \text { days }\end{array}$ \\
\hline Wang, 1999 [10] & 1 & $60 / \mathrm{M}$ & $\begin{array}{c}\text { Headache and } \\
\text { fever }\end{array}$ & $\begin{array}{c}\text { GB20, GB21, } \\
\text { SJ5 }\end{array}$ & Tetanus & Not specified & Dead \\
\hline Chen et al., 2008 [61] & 1 & $62 / \mathrm{F}$ & RA & Knee & Tetanus & Illegal treatment & Dead \\
\hline
\end{tabular}

hematoma due to acquired hemophilia B which improved after treatment [73], and lower limb (1) [74].

3.10. Complications Caused by Broken Needles (Table 9). Seven cases of accidents due to broken or bent needles were identified (Table 9). Five recovered after the surgery [7579 ], and 2 cases of bent needles were slowly pushed out by acupuncturists $[6,80]$.

3.11. Other Complications Associated with Acupuncture (Table 10). A total of 15 other complications associated with acu-puncture were reported (Table 10): aphonia [81], hoarseness [82], allergy to electroacupuncture [83] and metal [84], epilepsy [85, 86], fever [87], cough [88], thirst [88], infusion reaction [89], hyperventilation syndrome [90], and aggravation of fatigue [91]. Of them, 14 cases recovered completely and 1 improved.
One patient was not allergic after several acupuncture treatments, but systemic allergy occurred after EA treatment [65]. Among three patients with acupuncture-induced epilepsy, only one had a history of epilepsy $[85,86]$.

\section{Discussion}

The studies about safety of acupuncture are gradually increasing. One study protocol of a randomized controlled trial is efficacy and safety of acupuncture for chronic dizziness [92]. This trial's aim is to get result that acupuncture has good efficacy and without adverse effect for chronic dizziness. We hope it is success.

Some studies that researched acupuncture as an alternative means for pediatric diseases found that it is safe, feasible, and acceptable [93-99]. One study explored acupuncture as an effective therapy of pain relief for children and adolescents after tonsillectomy [100]. Severe throat pain can result from 
TABLE 8: Hemorrhage.

\begin{tabular}{|c|c|c|c|c|c|c|c|}
\hline $\begin{array}{l}\text { Author/year } \\
\text { (reference) }\end{array}$ & Cases & Age/sex & Disease treated & Punctured site & Complication & Practitioner & Follow-up \\
\hline Yang, 1986 [6] & 1 & $28 / \mathrm{F}$ & $\begin{array}{c}\text { Chronic } \\
\text { conjunctivitis }\end{array}$ & BL1 & Eye hematoma & Acupuncturist & $\begin{array}{l}\text { Recovered } \\
(14 \mathrm{f})\end{array}$ \\
\hline Li, 1989 [67] & 1 & $62 / F$ & Teratoma of ovary & Hypogastrium & $\begin{array}{l}\text { Extraperitoneal } \\
\text { hematoma }\end{array}$ & Roving doctor & $\begin{array}{c}\text { Recovered } \\
\text { after surgery }\end{array}$ \\
\hline Cai, 1991 [68] & 1 & $47 / \mathrm{M}$ & Neck mass & Neck & $\begin{array}{c}\text { Thyroid } \\
\text { intracapsular } \\
\text { hemorrhage with } \\
\text { apnea }\end{array}$ & Not specified & $\begin{array}{l}\text { Recovered } \\
\text { after surgery } \\
(10 \mathrm{~d})\end{array}$ \\
\hline Han, 1994 [69] & 1 & $56 / \mathrm{M}$ & Stroke & $\mathrm{RN} 23$ & $\begin{array}{l}\text { Sublingual } \\
\text { hematoma }\end{array}$ & Not specified & $\begin{array}{l}\text { Recovered } \\
\quad(1 \mathrm{wk})\end{array}$ \\
\hline $\begin{array}{l}\text { Zeng and Liu, } 1996 \\
\text { [71] }\end{array}$ & 1 & $50 / \mathrm{M}$ & Cough & ST9 & $\begin{array}{l}\text { Hematoma } \\
\text { compression } \\
\text { tracheal cause } \\
\text { apnea }\end{array}$ & $\begin{array}{l}\text { Unauthorized } \\
\text { acupuncturist }\end{array}$ & Dead \\
\hline Wang, 1996 [70] & 1 & $72 / \mathrm{M}$ & Cerebral infarction & Ex-HN20 & $\begin{array}{l}\text { Sublingual } \\
\text { hematoma }\end{array}$ & Not specified & $\begin{array}{l}\text { Recovered } \\
\quad(2 \mathrm{~d})\end{array}$ \\
\hline Gan, 2000 [66] & 1 & $46 / \mathrm{F}$ & Cold headache & Ex-HN05 & $\begin{array}{c}\text { Orbital } \\
\text { hemorrhage }\end{array}$ & Not specified & $\begin{array}{l}\text { Recovered } \\
\quad(1 \mathrm{mo})\end{array}$ \\
\hline Jiang, 2001 [72] & 1 & $68 / \mathrm{M}$ & $\begin{array}{l}\text { Hypertension, } \\
\text { stroke }\end{array}$ & LI4 & Hand hematoma & Acupuncturist & $\begin{array}{l}\text { Recovered } \\
\quad(7 \mathrm{~d})\end{array}$ \\
\hline Duan, 2007 [73] & 1 & $65 / F$ & $\begin{array}{l}\text { Neck, waist, and } \\
\text { leg pain }\end{array}$ & Hip & Buttock hematoma & Not specified & Improved \\
\hline $\begin{array}{l}\text { H. Liu and X. H. Liu, } \\
2007[74]\end{array}$ & 1 & $61 / \mathrm{M}$ & $\begin{array}{l}\text { Cerebral } \\
\text { thrombosis }\end{array}$ & SP6 & $\begin{array}{l}\text { Lower extremity } \\
\text { hematoma }\end{array}$ & Acupuncturist & $\begin{array}{l}\text { Recovered } \\
\quad(1 \mathrm{~d})\end{array}$ \\
\hline
\end{tabular}

TABLE 9: Complications caused by broken needles.

\begin{tabular}{|c|c|c|c|c|c|c|c|}
\hline $\begin{array}{l}\text { Author/year } \\
\text { (reference) }\end{array}$ & Cases & Age/sex & Disease treated & Punctured site & Complication & Practitioner & Follow-up \\
\hline Yang, 1986 [6] & 1 & $45 / \mathrm{M}$ & Flaccid paralysis & ST36, LI11 & Bent needle & Acupuncturist & Recovered \\
\hline $\begin{array}{l}\text { Lu and Teng, } 1994 \\
\text { [75] }\end{array}$ & 1 & $39 / \mathrm{M}$ & $\begin{array}{l}\text { Scapulohumeral } \\
\text { periarthritis }\end{array}$ & $\begin{array}{l}\text { Supraclavicular } \\
\text { fossa }\end{array}$ & $\begin{array}{c}\text { Hemopneumothorax } \\
\text { caused by broken } \\
\text { needle }\end{array}$ & Country doctor & $\begin{array}{l}\text { Recovered } \\
\text { after surgery }\end{array}$ \\
\hline Wang, 2000 [80] & 1 & $54 / \mathrm{M}$ & $\begin{array}{c}\text { The left upper limb } \\
\text { dysfunction }\end{array}$ & Upperlimb & Sticking of needle & Not specified & Recovered \\
\hline Geng, 2005 [76] & 1 & $58 / \mathrm{M}$ & $\begin{array}{l}\text { Chronic bronchitis } \\
\text { and emphysema }\end{array}$ & LU1 & $\begin{array}{l}\text { Damage of arteria } \\
\text { coronaria and cardiac } \\
\text { tamponade caused by } \\
\text { embedded needle }\end{array}$ & Self & $\begin{array}{l}\text { Recovered } \\
\text { after surgery }\end{array}$ \\
\hline Quan, 2008 [77] & 1 & $43 / \mathrm{F}$ & Gastric disease & Xiphoid & $\begin{array}{l}\text { Palpitation and } \\
\text { paroxysmal pricking } \\
\text { pain caused by } \\
\text { broken needle }\end{array}$ & Not specified & $\begin{array}{c}\text { Recovered } \\
\text { after surgery }\end{array}$ \\
\hline $\begin{array}{l}\text { Liu and Yu, } 2010 \\
{[78]}\end{array}$ & 1 & $45 / \mathrm{F}$ & $\begin{array}{c}\text { Multiple injuries } \\
\text { by traffic }\end{array}$ & $\mathrm{RN} 23$ & $\begin{array}{c}\text { Pulmonary bulla } \\
\text { caused by embedded } \\
\text { needle }\end{array}$ & Not specified & $\begin{array}{c}\text { Recovered } \\
\text { after surgery }\end{array}$ \\
\hline Cheng, 2010 [79] & 1 & $55 / \mathrm{M}$ & Lumbago & Lower limb & Broken needle & Not specified & $\begin{array}{l}\text { Recovered } \\
\text { after surgery }\end{array}$ \\
\hline
\end{tabular}

tonsillectomy and last up to 10 days in children. Codeine elixir has long been used for pain relief but has recently been banned by the Food and Drug Administration due to a recently recognized risk of death. This study suggested that acupuncture decreases perceived pain in children and adolescents after tonsillectomy. These data, combined with the cost effectiveness, safety, and ease of administering acupuncture, suggest that further studies exploring the effectiveness of acupuncture in juveniles after tonsillectomy are merited.

One research evaluated the feasibility of delivering acupuncture in an emergency department (ED) to patients presenting with pain and/or nausea [101]. The acupuncture 
TABLE 10: Other complications associated with acupuncture.

\begin{tabular}{|c|c|c|c|c|c|c|c|}
\hline $\begin{array}{l}\text { Author/year } \\
\text { (reference) }\end{array}$ & Cases & Age/sex & Disease treated & Acupoint & Complication & Practitioner & Follow-up \\
\hline \multirow[t]{2}{*}{$\begin{array}{l}\text { Wang and Lan, } \\
1980 \text { [81] }\end{array}$} & \multirow[t]{2}{*}{2} & $46 / \mathrm{F}$ & $\begin{array}{c}\text { Intercostal } \\
\text { neuralgia }\end{array}$ & \multirow[t]{2}{*}{ PC6 } & \multirow[t]{2}{*}{ Aphonia } & \multirow[t]{2}{*}{ Not specified } & \multirow[t]{2}{*}{$\begin{array}{l}\text { Recovered } \\
\quad(3 \mathrm{~d})\end{array}$} \\
\hline & & $36 / \mathrm{F}$ & Obstinate hiccup & & & & \\
\hline $\begin{array}{l}\text { Zhou et al., } 2005 \\
\text { [82] }\end{array}$ & 1 & $43 / \mathrm{F}$ & Neck pain & Ex-B05 & Hoarseness & Acupuncturist & $\begin{array}{l}\text { Recovered } \\
(10 \mathrm{~min})\end{array}$ \\
\hline Peng, 1982 [83] & 1 & $\begin{array}{c}54 / \text { not } \\
\text { specified }\end{array}$ & $\begin{array}{c}\text { Scapulohumeral } \\
\text { periarthritis }\end{array}$ & $\begin{array}{c}\text { Ex-UE01, } \\
\text { GB21, LI11, SI5 }\end{array}$ & $\begin{array}{l}\text { Allergy to } \\
\text { electroacupuncture }\end{array}$ & Acupuncturist & $\begin{array}{l}\text { Recovered } \\
(10 \mathrm{~min})\end{array}$ \\
\hline \multirow{2}{*}{$\begin{array}{l}\text { Gao and Zheng, } \\
2008[84]\end{array}$} & \multirow[b]{2}{*}{2} & $72 / \mathrm{M}$ & $\begin{array}{l}\text { Nerve root cervical } \\
\text { spondylopathy }\end{array}$ & EX-B2 & \multirow[b]{2}{*}{ Allergy to metal } & \multirow[b]{2}{*}{ Not specified } & $\begin{array}{l}\text { Recovered } \\
\quad(1 \mathrm{wk})\end{array}$ \\
\hline & & $49 / \mathrm{F}$ & $\begin{array}{c}\text { Cervical type } \\
\text { cervical } \\
\text { spondylopathy }\end{array}$ & EX-B2 & & & $\begin{array}{l}\text { Recovered } \\
\quad(5 \mathrm{~d})\end{array}$ \\
\hline Wang, 2004 [85] & 1 & $35 / \mathrm{M}$ & Soft tissue injury & Ashi point & Epilepsy & Acupuncturist & Recovered \\
\hline \multirow{2}{*}{ Dai, 2012 [86] } & \multirow[b]{2}{*}{2} & $45 / \mathrm{M}$ & Epilepsy & \multirow[b]{2}{*}{ Not specified } & \multirow[b]{2}{*}{ Epilepsy } & \multirow[b]{2}{*}{ Acupuncturist } & $\begin{array}{l}\text { Recovered } \\
\quad(2 \mathrm{~min})\end{array}$ \\
\hline & & $53 / \mathrm{M}$ & $\begin{array}{c}\text { Cervical } \\
\text { spondylosis }\end{array}$ & & & & $\begin{array}{l}\text { Recovered } \\
\quad(1 \mathrm{~min})\end{array}$ \\
\hline $\mathrm{Li}, 2000[87]$ & 1 & $52 / \mathrm{M}$ & $\begin{array}{l}\text { Cerebral } \\
\text { concussion }\end{array}$ & $\begin{array}{l}\text { DU20, GB20, } \\
\text { GB30, GB39, } \\
\text { LI4, LI11, ST36 }\end{array}$ & Fever & Acupuncturist & Recovered \\
\hline \multirow[t]{2}{*}{ Shang, 2006 [88] } & \multirow[t]{2}{*}{2} & $65 / F$ & Facial neuritis & $\begin{array}{l}\text { GB14, BL2, } \\
\text { ST2, SI18, } \\
\text { RN24, LI4, } \\
\text { ST36, LR3 }\end{array}$ & Cough & Acupuncturist & $\begin{array}{l}\text { Recovered } \\
\quad(2 \mathrm{~min})\end{array}$ \\
\hline & & $46 / \mathrm{F}$ & Obesity & $\begin{array}{l}\text { ST25, ST36, } \\
\text { SP15, RN6, } \\
\text { LI11, SJ6, SP9, } \\
\text { ST40 }\end{array}$ & Thirsty & Acupuncturist & Recovered \\
\hline $\begin{array}{l}\text { Quan and Jiang, } \\
2008 \text { [89] }\end{array}$ & 1 & $45 / \mathrm{F}$ & RA & Local points & Infusion reaction & Acupuncturist & $\begin{array}{l}\text { Recovered } \\
\quad(2 \mathrm{~h})\end{array}$ \\
\hline Fang, $2010[90]$ & 1 & $35 / \mathrm{F}$ & Cervical pain & GB20, EX-B2 & $\begin{array}{l}\text { Hyperventilation } \\
\text { syndrome }\end{array}$ & Acupuncturist & $\begin{array}{l}\text { Recovered } \\
(15 \mathrm{~min})\end{array}$ \\
\hline Wang, 2010 [91] & 1 & $46 / \mathrm{F}$ & $\begin{array}{l}\text { Nasopharyngeal } \\
\text { carcinoma } \\
\text { radiation sequela } \\
\text { with fatigue }\end{array}$ & $\begin{array}{l}\text { LI11, LI4, ST36, } \\
\text { SP6, KI3; LU7, } \\
\text { SI6, SJ3 }\end{array}$ & Aggravation of fatigue & Acupuncturist & Improved \\
\hline
\end{tabular}

group comprised 200 patients who received usual medical care and acupuncture; the usual care group comprised 200 patients with retrospective data closely matched from ED electronic health records. The results confirmed that acupuncture in the ED appears safe and acceptable for patients with pain and/or nausea. Further high-quality, sufficiently powered randomized studies evaluating the costeffectiveness and efficacy of the add-on effect of acupuncture are recommended.

Some reports confirmed that acupuncture for pregnant women is safe and effective [102-109]. For example, one reported a complete recovery from Bell's palsy (BP) of a 27year-old woman, 27-week pregnant, after 2 weeks of acupuncture treatment. Prior to treatment, her House-Brackmann facial nerve grading system (HBS) was II, Nottingham facial nerve grading system was $50.88 \%$, and the Facial Disability Indexes (FDI) were 90. After 2 weeks, her symptoms had disappeared, her face was restored to normal, HBS was I, Nottingham was $96.46 \%$, and FDIP was 100 . These results suggest that acupuncture may be a safe, alternative treatment for BP in pregnancy [110]. Another study described patients' experience of acupuncture treatment in low back and pelvic pain during pregnancy. Women received acupuncture treatment from gestational week 20 or week 26, for a period of 6 weeks divided into eight sessions of 30 minutes each. The results of Pain-O-Meter and visual analogue scale (POM-VAS), ShortForm McGill Questionnaire (SF-MPQ), and Short-form-36 health survey (SF-36) showed a relief of pain. Telephone interviews confirmed that expectations of treatment were fulfilled. The authors suggested that it may be advantageous 
to begin acupuncture therapy later in pregnancy to maximize pain relief [111].

However, complications and adverse effects in medical practice always concern the public and the medical profession. Acupuncture has been used for several thousand years in China. Although it has been deemed a safe and reliable therapy, the rare adverse effects and complications should arouse concerns. During the 33 years from 1980 to 2013, about 182 cases of acupuncture-associated complications and adverse effects were reported in China, including 25 fatal cases. The frequency of acupuncture associated complications reported in China appears to be steady over time (see Figure 2).

As indicated in Table 1, the most frequent complication of acupuncture treatment is internal organ, tissue, or nerve injury. Of the 115 reported cases involving internal organ, tissue, or nerve injury, 30 (26.08\%) were pneumothorax, 37 concerned the central nervous system $(32.17 \%)$, others included injury in peripheral nerve, organ, and other tissues. Based on our research, one major cause of direct thrusted injuries to organ, tissue, or nerve is the lack of knowledge about anatomy and other systems. In 1980s, the acupuncturists or individuals in many country grassroot regions performed acupuncture because of low cost and convenience, but the deficient knowledge on anatomy led to many cases of pneumothorax and subarachnoid hemorrhage, as well as injuries to abdomen organs, heart, and peripheral nerves. With the increasing requirement for acupuncturists, these accidents decreased from the 1990s. Particularly, the frequency of pneumothorax and central nervous system injury appears to be on the decline since the 2000s (see Figure 3). This may be because the government has demanded that the acupuncturists should have licenses and formal education background if they practice in clinic in recent years. They should also undergo short time training every year. All these make the acupuncture technique become more and more standardized so that the accidents of pneumothorax and central nervous system injury are reduced. However, there is a possibility that we lack the accident reporting system so that the incidences were underreported.

We put forward suggestions for the medical system in order to avoid more accidental injury on organ, such as enhancing training on anatomy for acupuncturists; setting up more continuation courses on the safety of acupuncture practice for acupuncturists; establishing a reporting system on the incidents of acupuncture adverse effect; and safety courses and certificates should be required in order to obtain the license of acupuncture in China.

The acupuncturists (1) should avoid important organs and tissues during selection of acupoints and reposition if the patient changes body position; (2) do not distract attention during treatment and do not move the patient after acupuncture so as to avoid accidents; for unconscious patients unable to cooperate, shallow needling or not retaining needle is preferred, and the process of treatment should be strictly monitored; (3) inquire detailed medical history and carefully determine the needling depth for patients with emphysema or hemorrhagic disease. Moreover, traditional medicine holds that acupuncture should be performed to bring about the

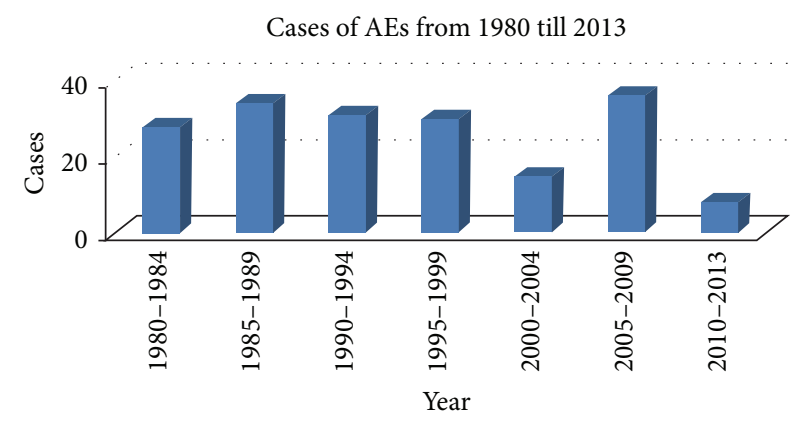

Figure 2: Distribution of cases of acupuncture-associated complications reported from 1980 to 2013 .

Cases of pneumothorax and central nervous system injury from 1980 till 2013

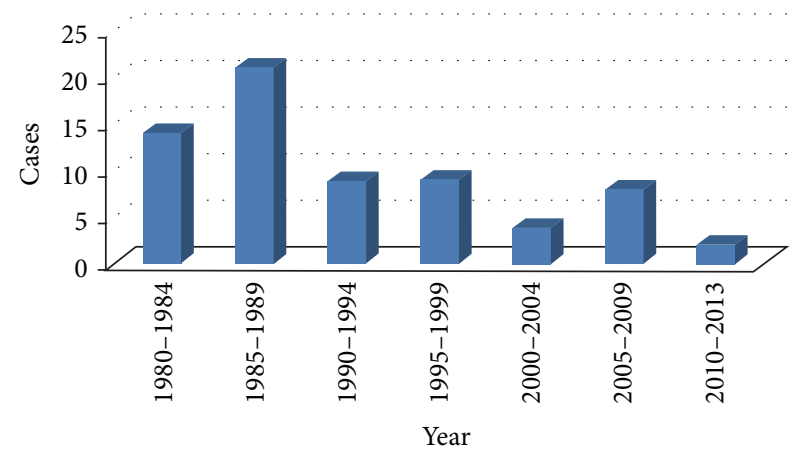

Figure 3: Distribution of cases of pneumothorax and central nervous system injury from 1980 to 2013.

desired sensations of "sour, numb, heavy, and swelling." Many acupuncturists and patients think that a stronger sensation of needling will bring about better therapeutic effects, but excessively violent operation will also cause accidents. The 2 cases of irritable stomach bleeding due to excessive irritation $[35,36]$ and the 1 case of femoral neck fracture due to myospasm [45] are typical examples and should alert clinicians. In case of suspected acupuncture-induced injury, the doctor should prolong the time of observation and warn for prompt treatment.

Syncope is also a common acupuncture-induced accident. The hungry, thirsty, drunken, or nervous patients should be asked to eat, drink, or rest for half an hour before treatment and calm down. They should be observed for a moment during and after treatment to avoid syncope. Once syncope occurs, needles should be pulled out immediately, sugar water should be provided, and the patient should lie down with head low; if the symptom becomes severe, take appropriate treatment. Two patients had severe adverse reaction like shock, with the clinical manifestation, including loss of consciousness, respiratory arrest, and carotid pulselessness. After doing CPR, both of them recovered [54, 55]. Reviewing medical history, one was found to have the similar experience several years ago [55]. Therefore, acupuncturists 
are required to inquire patients' medical history carefully and learn to deal with emergencies.

Acupuncture infection usually occurs in rural grassroot health centers with low awareness of hygiene, but accidents will be largely controlled along with the use of disposable needles and the popularization of health knowledge. Moreover, tetanus is still an adverse event that should be strictly prevented, and once it occurs, it will cause a high mortality rate. Along with the increasing incidence of diabetes, for patients with poorly controlled blood glucose [112], careful operation is required to avoid infection due to disunion of acupuncture-caused wounds.

The acupuncture-induced bleeding and hematoma are unavoidable; thus to reduce their incidence rates, acupuncturists should (1) get familiar with the anatomy of acupoints and avoid blood vessels during needle manipulation; (2) avoid manipulation methods such as lifting and thrusting when acupuncturing intraorbital acupoints; (3) appropriately extend the time of compression for patients with hypertension, arteriosclerosis, or inclination to hemorrhage and for women during the menstrual period. The acupunctureinduced hematoma is usually cold compressed within 24 hours and hot compressed after 24 hours. H. Liu and X. H. Liu [74] suggested pressing the local hematoma site heavily for a long time, which could immediately disperse the swelling, without leaving bruises. This method is recommended for other acupuncturists.

With a long history in China, acupuncture has been widely accepted and applied in people's daily life due to its exceptional therapeutic effects and low side effects. As early as 1980, WHO unveiled 43 kinds of diseases that can be treated with acupuncture. The number had increased to 107 in 2002 [113], from which we can see that acupuncture has been recognized by an increasing number of people and more research in this field is being undertaken. In countries where acupuncture is widely used, it is inevitable to encounter the occurrence of some side reactions in acupuncture therapies. However, the accident rate in acupuncture is relatively low. Although existing reports in China show no statistical data about acupuncture accidents, some studies conducted in large subject pools in Germany reveal some relevant information. It has been reported that Endres et al. [114] conducted a study about accidents in acupuncture therapies on 190,924 patients. The study showed an occurrence of 14,449 acupuncture accidents, which accounted for 7.57\% of the total subject pool. According to statistics conducted by Witt et al. [115] on 229,230 clinical acupuncture cases, there were 19,726 accidents, which occupied $8.6 \%$ of the total subject pool. Chinese literatures show that most of the acupuncture accidents are caused by acupuncturist's lack of corresponding techniques and nonstandard operations. Since 2005, the Chinese National Administration of Quality Supervision, Inspection and Quarantine and the Chinese National Standardization Management Committee have issued a total of 18 acupuncture technical operation specifications in two batches, including terms and definitions, operating procedures and requirements, operating methods, attentions, and contraindications. Among them, operating procedures and requirements specifically include the selection of needles, acupuncture points and acupuncture positions, environmental requirements, the disinfection of needles, selected acupuncture points and acupuncturist's hands, specific operation techniques, and after-treatments of wounds [116]. The acupuncture technical operation specification series covers a wide area and contains comprehensive and specific contents, but it still shows some deficiency in the popularization and implementation of acupuncture.

In conclusion, we recommend that the qualifying training of acupuncturists should be systemized and the clinical acupuncture operations be standardized in order to effectively prevent the occurrence of acupuncture accidents, enhance the influence of acupuncture, and further popularize acupuncture to the rest of the world. All this would mean a huge step towards modernization of acupuncture.

\section{Conflict of Interests}

The authors declare that there is no conflict of interests regarding the publication of this paper.

\section{Acknowledgments}

This work was supported partially by Grant no. 81102636 from the National Natural Science Foundation (NSFC) in China. In Austria, the work was supported by the Federal Ministries of Science, Research and Economy and of Health (project title "Evidence-Based High-Tech Acupuncture and Integrative Laser Medicine for Prevention and Early Intervention of Chronic Diseases"). The authors would like to thank Ms Ingrid Gaischek, M.S. (Medical University of Graz) for her valuable help concerning this paper.

\section{References}

[1] C. M. Pui, M. P. Bostrom, G. H. Westrich et al., "Increased complication rate following conversion total hip arthroplasty after cephalomedullary fixation for intertrochanteric hip fractures. A Multi-center Study," Journal of Arthroplasty, vol. 28, no. 8, pp. 45-47, 2013.

[2] L. Faunø, C. Rasmussen, K. K. Sloth, A. M. Sloth, and A. Tøttrup, "Low complication rate after stoma closure. Consultants attended $90 \%$ of the operations," Colorectal Disease, vol. 14, no. 8, pp. e499-e505, 2012.

[3] T. Umuroglu, K. Bostanci, D. T. Thomas, M. Yuksel, and F. Yilmaz Gogus, "Perioperative anesthetic and surgical complications of the nuss procedure," Journal of Cardiothoracic and Vascular Anesthesia, vol. 27, no. 3, pp. 436-440, 2013.

[4] L. Lao, G. R. Hamilton, J. Fu, and B. M. Berman, "Is acupuncture safe? A systematic review of case reports," Alternative Therapies in Health and Medicine, vol. 9, no. 1, pp. 72-83, 2003.

[5] S. F. Xu, L. Z. Wang, E. Cooper et al., "Adverse events of acupuncture: a systematic review of case reports," EvidenceBased Complementary and Alternative Medicine, vol. 2013, Article ID 581203, 15 pages, 2013.

[6] Y. D. Yang, "Clinical examples of adverse events of acupuncture ," Shanxi Journal of Traditional Chinese Medicine, vol. 7, no. 7, p. 319, 1986. 
[7] Y. L. Wang, "A case about death because of pneumothorax caused by needling LI17-tianding," Journal of Forensic Medicine, vol. 15, no. 1, pp. 47-48, 1999.

[8] Y. D. Yang, R. Shi, and S. H. Ge, "Presentation about one case of subarachnoid hemorrhage caused by needling," Liaoning Journal of Traditional Chinese Medicine, vol. 8, p. 37, 1985.

[9] Q. L. Song and T. Y. Wu, "Report of three cases about malpractice of acupuncture," Clinical Misdiagnosis \& Mistherapy, vol. 14, no. 1, p. 73, 2001.

[10] K. F. Wang, "Some thoughts about acupuncture accidents," Journal of Clinical Acupuncture and Moxibustion, vol. 15, no. 5, pp. 57-58, 1999.

[11] A. Chen, "Analysis on four cases about acupuncture accident," Shanghai Journal of Acupuncture and Moxibustion, vol. 28, no. 2, pp. 112-113, 2009.

[12] J. Mi, "A case about cerebral hemorrhage caused by acupuncture," Heilongjiang Journal of Traditional Chinese Medicine, no. 2, p. 34, 1993.

[13] S. X. Li, H. Wen, and X. H. Yao, "A case about death caused by leukemia acute intracerebral hemorrhage with acupuncture damage," Chinese Journal of Forensic Medicine, vol. 26, no. 4, pp. 334-335, 2011.

[14] X. J. Liu, "Four cases accidents of schizophrenia treating by acupuncture," Chinese Journal of Nervous and Mental Diseases, vol. 5, p. 317, 1981.

[15] X. L. Tang and Q. P. Fang, "Aggravation of infranuclear facial palsy induced by strong stimulation of electro-acupuncture," People's Military Surgeon, no. 6, p. 69, 1986.

[16] X. F. Nie and A. J. Zhou, "Analysis on the reason of respiratory and cardiac arrest induced by needling in SJ17-yifeng," Hubei Journal of Traditional Chinese Medicine, vol. 5, p. 39, 1990.

[17] B. X. Yan, "One case of phrenic nerve damage caused by needling in brachial plexus," Shaanxi Journal of Traditional Chinese Medicine, vol. 15, no. 11, p. 513, 1994.

[18] G. Yang and S. L. Wang, "Two reports about blindness caused by acupuncture," Tianjin Journal of Traditional Chinese Medicine, vol. 13, no. 1, p. 6, 1996.

[19] L. H. Xu and A. H. Liu, "One case about oculomotor nerve damage caused by acupuncture," Journal of Injuries and Occupational Diseases of the Eye with Ophthalmic Surgeries, vol. 19, no. 3, article 226, 1997.

[20] R. H. Huang and S. H. Wei, "A case of eye damage in treating trigeminal nerve pain with electro-acupuncture," Chinese Journal of Ocular Trauma and Occupational Eye Disease (with Ophthalmic Surgery), vol. 19, no. 4, p. 307, 1997.

[21] J. W. Ruan, S. M. Li, M. Wen, Z. D. Rao, and Y. H. Hu, "Analysis on adverse effects of acupuncture in clinical practices," Chinese Acupuncture \& Moxibustion, vol. 29, no. 11, pp. 939-942, 2009.

[22] J. Zhu, "A case about death caused by thoracic aorta hemorrhage through needling in LR14-qimen," Shanghai Journal of Acupuncture and Moxibustion, no. 2, p. 31, 1990.

[23] G. W. Shi, "A case about death caused by aortic rupture through needling in the chest," Chinese Journal of Forensic Medicine, vol. 8, no. 4, pp. 237-242, 1993.

[24] J. Z. Xie and W. Z. Lin, "A case about death because of cardiac trauma caused by acupuncture," Chinese Journal of Integrated Traditional and Western Medicine in Intensive and Critical Care, vol. 10, no. 1, p. 51, 2003.

[25] Y. Z. Luo, L. Dong, H. S. Yuan, and Q. L. Xiang, "A case about delayed cardiac tamponade caused by acupuncture," Chinese Journal of Clinical Thoracic and Cardiovascular Surgery, vol. 13, no. 5, p. 343, 2006.
[26] J. H. Zhang and X. R. Dong, "A case about death because of cardiac rupture caused by acupuncture," Journal of Forensic Medicine, no. 2, p. 49, 2006.

[27] W. T. Zhu, X. S. Li, Y. T. Zhang, and W. Li, "A case about death because of cardiac rupture caused by acupuncture," Chinese Journal of Forensic Medicine, vol. 24, no. 4, p. 312, 2008.

[28] S. T. Yang, "A case about chylothorax caused by needling in BL 13-Feishu," Journal of Clinical Acupuncture and Moxibustion, no. 4, p. 19, 1991.

[29] Q. L. Duan and S. R. Wang, "Lesson learn from three cases about severe complications caused by acupuncture," Hebei Journal of Traditional Chinese Medicine, no. 1, pp. 36-37, 1984.

[30] B. S. Zheng and M. L. Zhao, "Lessons learn from two cases about perforation of gallbladder and biliary peritonitis caused by acupuncture," Chinese Journal for Clinicians, no. 3, p. 44, 1983.

[31] M. H. Deng, "Report of one case about gallbladder perforation caused by needling in ST21-liangmen," Journal of Sichuan of Traditional Chinese Medicine, vol. 4, p. 13, 1985.

[32] X. F. Bai, "One case about perforation of gallbladder and biliary peritonitis caused by acupuncture," Inner Mongolia Medical Journal, vol. 11, no. 2, article 80, 1991.

[33] J. H. Zhang, "Four reports of peritonitis caused by needling in the abdomen," Chinese Journal of Rural Medicine and Pharmacy, vol. 4, no. 6, pp. 20-21, 1997.

[34] X. T. Xiao, "Lessons learned from one case about gastric perforation caused by acupuncture," Chinese Journal for Clinicians, no. 1, p. 9, 1985.

[35] L. H. Huang, "A case of gastric ulcer with perforation caused by needling in ST36-zusanli," Journal of Clinical Acupuncture and Moxibustion, vol. 15, no. 10, p. 43, 1999.

[36] T. Tang, Q. Li, and J. X. Gao, "A case about clinical acupuncture accident," Chinese Acupuncture \& Moxibustion, vol. 26, no. 6, pp. 459-460, 2006.

[37] W. G. Liu, L. J. Duan, and D. T. Sun, "Report of one case about intestinal obstruction caused by needling in ST25-tianshu," Chinese Journal of Integrated Traditional and Western Medicine, vol. 2, no. 3, p. 147, 1992.

[38] C. Y. Zhang, S. M. Zhen, and Y. F. Pei, "Report of five cases about visceral injury caused by acupuncture," Heilongjiang Journal of Traditional Chinese Medicine, no. 1, p. 38, 1992.

[39] S. M. Chen, "One case about cervical common carotid aneurysm caused by acupuncture," Journal of Traditional Chinese Medicine, no. 7, p. 49, 1980.

[40] Z. Z. Wang, "A case of shock induced by acupuncturing in carotid sinus," Chinese Journal of Practical Surgery, vol. 7, no. 2, p. $66,1987$.

[41] J. Zhu, "A case about death because of asphyxia caused by needling in RN 22-tiantu," Jiangsu Journal of Traditional Chinese Medicine, vol. 2, article 28, 1986.

[42] L. W. Gao, "A case about dyspnea caused by needling LI18-futu," China Journal of Traditional Chinese Medicine and Pharmacy, no. 2, p. 50, 1989.

[43] B. S. Liu, Z. X. Li, and F. Lei, "A case about amotio retinae caused by acupuncture," Chinese Journal of Ocular Trauma and Occupational Eye Disease(With Ophthalmic Surgery), vol. 1, pp. 43-44, 1988.

[44] S. F. Wang, "Adverse reaction about two cases caused by needling in Ex-HN5-taiyang with improper technique," Chinese Journal of Practical Meicine, no. 3, p. 135, 1982.

[45] Y. M. Li and Z. Q. Zhou, "Report of one case about femoral neck fracture induced by electro-acupuncture," Chinese Journal of Nervous and Mental Diseases, vol. 3, p. 153, 1980. 
[46] Y. T. Liang and L. F. Song, "One case of flexor hallucis muscle and digitorum longus muscle scar contracture caused by repeated acupuncture," People's Military Surgeon, no. 5, pp. 79-80, 1984.

[47] Y. Z. Chen, "Analysis on one case of nape spasm induced by electro-acupuncturing in GB20-fengchi and Ex-HN17-yiming," Journal of Clinical Acupuncture and Moxibustion, vol. 1, article 50, 1992.

[48] J. H. Wang, "A case of subluxation of wrist caused by improper electro-acupuncture," Guangxi Journal of Traditional Chinese Medicine, no. 1, p. 45, 1994.

[49] G. P. Shi and Z. M. Chen, "Analysis on three cases of adverse events caused by acupuncture," Journal of Clinical Acupuncture and Moxibustion, vol. 10, no. 5, p. 38, 1994.

[50] C. Y. Luo and H. Y. Huang, "Two cases of fasciculation induced by acupuncture," Chinese Acupuncture and Moxibustion, vol. 26, no. 6, p. 460, 2006.

[51] W. L. Shao, "Report of one case about syncope after drink," Journal of Nanjing University of Traditional Chinese Medicine, no. 4 , p. $41,1989$.

[52] C. B. Liu, "Report of four case about syncope induced by acupuncture," Journal of Clinical Acupuncture and Moxibustion, vol. 17, no. 3, article 51, 2001.

[53] L. Li, J. Y. Huang, and G. M. Lu, "Two cases of lag syncope induced by acupuncture," Chinese Acupuncture and Moxibustion, vol. 29, no. 9, p. 732, 2009.

[54] Z. H. Ma, "A case of sudden cardiac arrest induced by acupuncture," Hebei Journal of Traditional Chinese Medicine, vol. 27, no. 8, p. 589, 2005.

[55] Z. Y. Long, D. S. Chen, and L. P. Chen, "Analysis on cases of severe syncope during acupuncture treatment," Hubei Journal of Traditional Chinese Medicine, vol. 28, no. 9, p. 52, 2006.

[56] Z. Q. Wu, J. C. Lv, and H. F. Ma, "A case about ear-acupuncture treatment of abnormal sense after needling," Journal of Hebei Traditional Chinese Medicine and Pharmacology, vol. 16, no. 4, p. 32, 2001.

[57] B. S. Yang, Y. Wu, L. Y. Wang, and Y. X. Xu, "One case about tetanus caused by needling in Ex-UE19," Chinese Community Doctors, no. 2, p. 31, 1990.

[58] G. X. Liu, "One case about tetanus caused by acupuncture," The Journal of Medical Theory and Practice, vol. 4, no. 3, article 30, 1991.

[59] C. R. Liu, "One case about tetanus caused by acupuncture," Chinese Community Doctors, no. 4, p. 30, 1992.

[60] Y. E. Sun and Q. G. Hu, "A case about tetanus caused by acupuncture during the treatment for Facioplegia," Journal of Oral and Maxillofacial Surgery, vol. 7, no. 2, p. 108, 1997.

[61] Z. G. Chen, J. Zhang, J. J. Li, and D. Z. Jia, "One case about death because of tetanus caused by acupuncture," Forensic Science and Technology, no. 2, p. 74, 2008.

[62] D. X. Xie and S. W. Zong, "One lesson about abdominal metastatic hepatic hydatid caused by acupuncture," Chinese Journal for Clinicians, no. 3, p. 45, 1983.

[63] Y. S. Chen and L. Gao, "One case about encephalopyosis caused by needling on the head," People's Military Surgeon, vol. 4, article 58, 1995.

[64] Z. Zhou, "Report of three cases about tuberculous abscess on the body surface caused by acupuncture and moxibustion," Journal of Jiujiang University, no. 1, p. 47, 1999.

[65] F. Gao and Y. Z. Qi, "Report of one case about locally burn caused by electro-acupuncture," Shanghai Journal of Acupuncture and Moxibustion, vol. 4, p. 15, 1989.
[66] Z. Z. Gan, "A case about massive orbital hemorrhage caused by neeldling in Ex-HN05," Chinese Journal of Ocular Trauma and Occupational Eye Disease, vol. 22, no. 3, p. 246, 2000.

[67] D. P. Li, "One case report of extraperitoneal hematoma casued by needling in the abdomen," Shaanxi Medical Journal, vol. 18, no. 11, pp. 53-54, 1989.

[68] X. J. Cai, "One case about apnea caused by thyroid intracapsular hemorrhage because of needling," Medical Journal of Chinese People’s Liberation Army, no. 6, p. 494, 1991.

[69] L. X. Han, "One case of sublingual fold hematoma caused by needling RN23-lianquan," Shanghai Journal of Acupuncture and Moxibustion, vol. 13, no. 3, p. 107, 1994.

[70] N. R. Wang, "A case about sublingual hematoma caused by needling in Ex-HN20," Journal of Clinical Acupuncture and Moxibustion, vol. 1, no. 8, p. 8, 1996.

[71] X. F. Zeng and Z. Y. Liu, "A case about death caused by needling in ST 9-renying," in Proceedings of the 5th National Clinical Forensic Medicine Symposium of Chinese forensic Medicine Association, p. 408, 1996.

[72] Y. Jiang, "Analysis on one case of hematoma induced by hegustimulate in LI4-hegu," Journal of Clinical Acupuncture and Moxibustion, vol. 17, no. 5, p. 46, 2001.

[73] H. Duan, "One case report of hematoma after acupuncture in acquired hemophilia B," Practical Journal of Clinical Medicine, vol. 4, no. 2, p. 32, 2007.

[74] H. Liu and X. H. Liu, "Prevention and treatment of haematoma in acupuncture," Chinese Journal of Misdiagnostics, vol. 7, no. 29, p. 71, 2007.

[75] Y. D. Lu and Q. S. Teng, "A case about hemopneumothorax caused by breaking needle in the chest during acupuncture," Medical Journal of Chinese People's Liberation Army, vol. 19, no. 5, article 381, 1994.

[76] J. Q. Geng, "A case about successful rescue of acute pericardial tamponade caused by acupuncturing coronary artery," Journal of Clinical Cardiology, vol. 21, no. 7, p. 392, 2005.

[77] S. S. Quan, "A case of metal foreign body in the heart," Journal of Medical Science Yanbian University, vol. 31, no. 4, p. 304, 2008.

[78] W. H. Liu and G. X. Yu, "A case about pulmonary bulla caused by acupuncture," Chinese Acupuncture and Moxibustion, vol. 30, no. 8, p. 674, 2010.

[79] R. S. Cheng, "One case about broken needle caused by wristankle acupuncture," Shanghai Journal of Acupuncture and Moxibustion, vol. 29, no. 7, p. 463, 2010.

[80] H. F. Wang, "One case of sticking and damage of needles in acupuncture treatment of trauma patient," Journal of Clinical Acupuncture and Moxibustion, vol. 16, no. 11, p. 42, 2000.

[81] S. F. Wang and Y. H. Lan, "Acute aphonia caused by acupuncture in PC6-neiguan," New Chinese Medicine, vol. 11, no. 11, pp. 587$588,1980$.

[82] L. Z. Zhou, D. G. Zhao, and L. Peng, "Some thoughts of hoarseness casued by acupuncture," Shanghai Journal of Acupuncture and Moxibustion, vol. 24, no. 1, p. 27, 2005.

[83] Y. G. Peng, "Report of one case about allergy induced by electroacupuncture," Jiangxi Journal of Traditional Chinese Medicine, vol. 3, article 19, 1982.

[84] Q. S. Gao and Y. J. Zheng, "Two cases about acupuncture allergy," Chinese Acupuncture and Moxibustion, vol. 28, no. 11, p. $818,2008$.

[85] L. Wang, "One case of epilepsy seizures caused by acupuncture," Chinese Acupuncture Moxibustion, vol. 24, p. 96, 2004. 
[86] S. Dai, “Two cases of epilepsy caused by acupuncture," Jiangxi Journal of Traditional Chinese Medicine, vol. 43, no. 6, p. 38, 2012.

[87] J. S. Li, "A case about fever induced by acupuncture," Chinese Acupuncture and Moxibustion, vol. 20, no. 10, p. 639, 2000.

[88] Y. T. Shang, "Two adverse reactions induced by acupuncture," Journal of New Chinese Medicine, vol. 38, no. 11, article 76, 2006.

[89] G. S. Quan and L. Jiang, "One case report of infusion reaction caused by acupuncture," Jilin Medical Journal, vol. 29, no. 1, p. 79, 2008.

[90] W. Fang, "Case report of hyperventilation syndrome induced by acupuncture," Journal of Emergency in Traditional Chinese Medicine, vol. 19, no. 3, pp. 486-488, 2010.

[91] C. H. Wang, "A case of adverse reaction induced by acupuncture when treating sequelae of radiotherapy in nasopharyngeal carcinoma," Shanghai Journal of Acupuncture and Moxibustion, vol. 29, no. 8, pp. 536-537, 2010.

[92] Z. Xue, C. Z. Liu, G. X. Shi et al., "Efficacy and safety of acupuncture for chronic dizziness: study protocol for a randomized controlled trial," Trials, vol. 14, p. 429, 2013.

[93] D. C. A. Ferreira, A. De Rossi, C. P. Torres et al., "Effect of laser acupuncture and auricular acupressure in a child with trismus as a sequela of medulloblastoma," Acupuncture in Medicine, vol. 32, no. 2, pp. 190-193, 2014

[94] G. Karlson and P. Bennicke, "Acupuncture in asthmatic children: a prospective, randomized, controlled clinical trial of efficacy," Alternative Therapies in Health and Medicine, vol. 19, no. 4, pp. 13-19, 2013.

[95] M. Y. Elseify, N. H. Mohammed, A. A. Alsharkawy, and M. E. Elseoudy, "Laser acupuncture in treatment of childhood bronchial asthma," Journal of Complementary and Integrative Medicine, vol. 10, no. 1, pp. 199-203, 2013.

[96] Y. Moustafa, A. N. Kassab, J. el Sharnoubi, and H. Yehia, "Comparative study in the management of allergic rhinitis in children using LED phototherapy and laser acupuncture," International Journal of Pediatric Otorhinolaryngology, vol. 77, no. 5, pp. 658-665, 2013.

[97] E. F. Anders, A. Findeisen, A. Nowak, M. Rüdiger, and T. I. Usichenko, "Acupuncture for treatment of hospital-induced constipation in children: a retrospective case series study," Acupuncture in Medicine, vol. 30, no. 4, pp. 258-260, 2012.

[98] X. L. Ni, Y. N. Xie, Q. Wang et al., "Cardioprotective effect of transcutaneous electric acupoint stimulation in the pediatric cardiac patients: a randomized controlled clinical trial," Paediatric Anaesthesia, vol. 22, no. 8, pp. 805-811, 2012.

[99] M. Reinthal, I. Lund, D. Ullman, and T. Lundeberg, "Gastrointestinal symptoms of infantile colic and their change after light needling of acupuncture: a case series study of 913 infants," Chinese Medicine, vol. 6, no. 1, article 28, 2011.

[100] J. W. Ochi, "Acupuncture instead of codeine for tonsillectomy pain in children," International Journal of Pediatric Otorhinolaryngology, vol. 77, no. 12, pp. 2058-2062, 2013.

[101] A. L. Zhang, S. J. Parker, D. M. Taylor, and C. C. Xue, "Acupuncture and standard emergency department care for pain and/or nausea and its impact on emergency care delivery: a feasibility study," Acupuncture in Medicine, 2014.

[102] G. P. C. Gribel, L. G. Coca-Velarde, and R. A. Moreira de Sá, "Electroacupuncture for cervical ripening prior to labor induction: a randomized clinical trial," Archives of Gynecology and Obstetrics, vol. 283, no. 6, pp. 1233-1238, 2011.

[103] J. B. G. da Silva, M. U. Nakamura, J. A. Cordeiro, L. Kulay, and R. Saidah, "Acupuncture for dyspepsia in pregnancy: a prospective, randomised, controlled study," Acupuncture in Medicine, vol. 27, no. 2, pp. 50-53, 2009.

[104] J. B. G. Da Silva, "Acupuncture for mild to moderate emotional complaints in pregnancy-a prospective, quasi-randomised, controlled study," Acupuncture in Medicine, vol. 25, no. 3, pp. 65-71, 2007.

[105] J. B. Guerreiro Da Silva, M. U. Nakamura, J. A. Cordeiro, and L. Kulay Jr., "Acupuncture for insomnia in pregnancy-a prospective, quasi-randomised, controlled study," Acupuncture in Medicine, vol. 23, no. 2, pp. 47-51, 2005.

[106] J. B. Guerreiro da Silva, M. U. Nakamura, J. A. Cordeiro, and L. Kulay Jr., "Acupuncture for low back pain in pregnancy-a prospective, quasi-randomised, controlled study," Acupuncture in Medicine, vol. 22, no. 2, pp. 60-67, 2004.

[107] N. Kvorning, C. Holmberg, L. Grennert, A. Aberg, and J. Akeson, "Acupuncture relieves pelvic and low-back pain in late pregnancy," Acta Obstetricia et Gynecologica Scandinavica, vol. 83, no. 3, pp. 246-250, 2004.

[108] K. Wedenberg, B. Moen, and Å. Norling, "A prospective randomized study comparing acupuncture with physiotherapy for low-back and pelvic pain in pregnancy," Acta Obstetricia et Gynecologica Scandinavica, vol. 79, no. 5, pp. 331-335, 2000.

[109] T. Rosen, M. de Veciana, H. S. Miller, L. Stewart, A. Rebarber, and R. N. Slotnick, "A randomized controlled trial of nerve stimulation for relief of nausea and vomiting in pregnancy," Obstetrics and Gynecology, vol. 102, no. 1, pp. 129-135, 2003.

[110] H. Lei, W. Wang, and G. Huang, "Acupuncture benefits a pregnant patient who has Bell's palsy: a case study," The Journal of Alternative and Complementary Medicine, vol. 16, no. 9, pp. 1011-1014, 2010.

[111] L. Ekdahl and K. Petersson, "Acupuncture treatment of pregnant women with low back and pelvic pain-an intervention study," Scandinavian Journal of Caring Sciences, vol. 24, no. 1, pp. 175-182, 2010.

[112] X. Y. Ding, S. Y. Liu, and J. Zhu, "One case about clinical acupuncture accident," Chinese Acupuncture \& Moxibustion, vol. 28, no. 11, pp. 817-818, 2008.

[113] World Health Organization, Acupuncture: Review and Analysis of Reports on Controlled Clinical Trials, World Health, 2002.

[114] H. G. Endres, A. Molsberger, M. Lungenhausen, and H. J. Trampisch, "An internal standard for verifying the accuracy of serious adverse event reporting: the example of an acupuncture study of 190,924 patients," European Journal of Medical Research, vol. 9, no. 12, pp. 545-551, 2004.

[115] C. M. Witt, D. Pach, B. Brinkhaus et al., "Safety of acupuncture: results of a prospective observational study with 229,230 patients and introduction of a medical information and consent form," Research in Complementary Medicine, vol. 16, no. 2, pp. 91-97, 2009.

[116] W. J. He, Y. Q. Li, X. Zhao et al., "Overview of the acupuncture safety standards at home and abroad," in Proceedings of Chinese Acupuncture Association Annual Meeting, pp. 62-67, 2011.

[117] D. G. Jiang, "Report of one case about pneumothorax caused by needling in RN15-jiuwei," Chongqing Medicine, no. 6, p. 47, 1980.

[118] Y. Z. Cai and Q. L. Wang, "A case about large hemothorax caused by deep needling," Chinese Journal of Medicine, no. 6, p. 54, 1982.

[119] P. D. Zheng and S. H. Pang, "One case about severe hemopneumothorax caused by acupuncture," Journal of Traditional Chinese Medicine, vol. 5, article 32, 1983.

[120] C. G. Gao, "Report of two cases about pneumothorax caused by acupuncture," The Journal of Practical Medicine, vol. 3, pp. 3839, 1984. 
[121] J. Y. Chang, "A case about large hemopneumothorax caused by needling in the back," Hebei Journal of Traditional Chinese Medicine, no. 3, p. 48, 1984.

[122] Z. C. Yan, "A case about severe pneumothorax caused by needling RN22-tiantu," New Chinese Medicine, vol. 16, no. 12, p. 655, 1985.

[123] Q. X. Hu, "A case about pneumothorax caused by improper acupuncture," Shanghai Journal of Acupuncture and Moxibustion, vol. 3, p. 34, 1986.

[124] S. P. Zhang, "A case about large hemopneumothorax caused by acupuncture," Shanghai Journal of Acupuncture and Moxibustion, vol. 6, no. 4, p. 21, 1986.

[125] B. R. Jin, "A case about pneumothorax caused by needling SI13quyuan," Shanghai Journal of Acupuncture and Moxibustion, no. 3, p. 45, 1987.

[126] B. Z. Song and J. S. Xu, "Report of one case about hemothorax caused by needling left GB21-jianjing," Journal of Anhui Traditional Chinese Medical College, vol. 6, no. 3, p. 45, 1987.

[127] K. Y. Ruan, K. J. Qi, and Z. C. Mou, "A case about hydropneumothorax caused by needling supraclavicular fossae," Journal of New Chinese Medicine, vol. 4, p. 33, 1992.

[128] B. Lu, "Report of one case about traumatic pneumothorax caused by needling too deep," Guizhou Medicine Journal, vol. 17, no. 2, p. 125, 1993.

[129] C. Xia, "Handling method and experience of accident occurred during acupuncture," Journal of Clinical Acupuncture and Moxibustion, no. 2-3, pp. 75-76, 1993.

[130] Y. W. Li and X. L. Chen, "A case about pneumothorax caused by acupuncture," Journal of Handan Medical College, vol. 10, no. 3, pp. 72-73, 1997.

[131] L. Ma and C. J. Zhang, "Clinical analysis of pneumothorax caused by acupuncture," Journal of Clinical Acupuncture and Moxibustion, vol. 13, no. 2, p. 40, 1997.

[132] M. X. Qin and W. H. Ao, "Pneumothorax caused by improper acupuncture and moxibustion," Clinical Misdiagnosis \& Mistherapy, vol. 16, no. 1, article 76, 2003.

[133] C. H. Zha, "Injury identification of one case about hemopneumothorax caused by acupuncturing left lung," Chinese Journal of Forensic Medicine, no. 21, pp. 75-76, 2006.

[134] J. Y. Gan, G. Q. Lei, and J. S. Zhu, "One case about hemopneumothorax and dyspnea caused by acupuncture," in Proceedings of the 9th National Clinical Forensic Medicine Symposium of Chinese forensic Medicine Association, pp. 58-59, 2006.

[135] S. H. Huo, Y. P. Tian, Q. L. Ma et al., "One case about cardiac trauma and pneumothorax caused by acupuncture," Chinese Journal of Critical Care Medicine, vol. 27, no. 10, p. 908, 2007.

[136] L. Y. Liu, "Analysis on one case of hemopneumothorax induced by acupuncture," Journal of Changchun University of Traditional Chinese Medicine, vol. 23, no. 3, p. 58, 2007.

[137] B. Z. Ma, "Report of one case about traumatic pneumothorax caused by acupuncture and moxibustion," Qinghai Medical Journal, vol. 37, no. 2, p. 41, 2007.

[138] J. Zhang and L. Zhao, "Analysis and treatment of pneumothorax caused by needling GB21-jianjing," Hubei Journal of Traditional Chinese Medicine, vol. 34, no. 11, pp. 60-61, 2012.

[139] S. X. Liu, "Subarachnoid hemorrhage caused by needling in GB20-fengchi," Shanxi Medical Journal, vol. 9, no. 6, article 53, 1980.

[140] L. P. Bao and C. T. Gao, "Report of three cases about subarachnoid hemorrhage induced by needling GB20-fengchi and DU15-yamen," Jilin Medical Journal, vol. 4, no. 3, pp. 45-53, 1983.
[141] Q. T. Chen and L. J. Wu, "Case about subarachnoid hemorrhage and medulla oblogata hemorrhage caused by needling in anmian point," Journal of Apoplexy and Nervous Diseases, vol. 2, no. 3, pp. 158-159, 1985.

[142] Y. Z. Chen and W. C. Huang, "One case of subarachnoid hemorrhage caused by needling in the nape," Shanxi Journal of Traditional Chinese Medicine, vol. 1, no. 1, pp. 47-48, 1985.

[143] Y. Su, H. X. Chen, C. C. Chen, and Z. J. Yang, "One case of subarachnoid hemorrhage caused by needling in Du15-yamen," Shanghai Journal of Acupuncture and Moxibustion, vol. 3, p. 22, 1985.

[144] B. R. Yu, "Subarachnoid hemorrhage caused by needling in the nape-with report of seven cases," Journal of Apoplexy and Nervous Diseases, vol. 3, no. 2, pp. 74-75, 1986.

[145] M. X. Chen, "One case of subarachnoid hemorrhage caused by needling in Du15-yamen," Chinese Journal of Practical Internal Medicine, vol. 7, no. 7, p. 355, 1987.

[146] S. C. Jiang and X. Q. Chen, "A case about death caused by needling in GB20-fengchi," Chinese Journal of Forensic Medicine, vol. 2, no. 2, pp. 116-117, 1987.

[147] J. W. Zhou, "A case about death caused by needling in GB20fengchi," Journal of Sichuan of Traditional Chinese Medicine, no. 10, p. 52, 1988.

[148] X. H. Mi, S. K. Li, and S. K. Jia, "One case of subarachnoid hemorrhage caused by needling in Du15-yamen," Shandong Journal of Traditional Chinese Medicine, vol. 8, no. 2, p. 22, 1989.

[149] B. C. Wu and Q. Z. Xu, "Report of one case about death caused by spinal cord injury through acupuncturing in acupoint near C3," Chinese Journal of Forensic Medicine, vol. 5, no. 1, pp. 32-33, 1990.

[150] F. Y. Liu, "One case of posterior fossa acute subdural hematoma caused by needling in GB20-fengchi," Medical Journal of Liaoning, vol. 6, no. 1, p. 25, 1992.

[151] T. Z. Jiang, W. D. Chen, B. Zhang, and R. M. Yang, "Report of two cases about subarachnoid hemorrhage caused by needling in the nape," Journal of Anhui Traditional Chinese Medicine College, vol. 15, no. 1, p. 48, 1996.

[152] M. Liu, S. P. Shen, H. Tong, and T. Wang, "Autopsy report of medulla oblongata hemorrhage caused by needling in DU16fengfu," in Proceedings of the 5th National Clinical Forensic Medicine Symposium of Chinese Forensic Medicine Association, p. 85, 1996.

[153] F. Bian, X. L. Zhang, and S. P. Tian, "A case about subarachnoid hemorrhage caused by needling in GB20-fengchi," Journal of Hebei North University (Medical Edition), vol. 14, no. 6, article 73, 1997.

[154] L. Wang, "A case about death caused by needling in cervical spinal cord," Forensic Science and Technology, no. 4, p. 20, 1999.

[155] Y. Q. Li, G. W. Chen, and X. C. Zeng, "A case about cervical spinal cord epidural hematoma caused by acupuncture," Chinese Journal of Rural Medicine and Pharmacy, vol. 10, no. 3, p. 20, 2003.

[156] L. J. Niu and R. W. Zhang, "A case about cisterna magna hemorrhage caused by acupuncture," Practical Journal of Medicine \& Pharmacy, vol. 23, no. 8, p. 932, 2006.

[157] L. F. Li, X. Cong, and Q. W. Jin, "Subarachnoid hemorrhage caused by needling in DU16-fengfu," Chinese Acupuncture \& Moxibustion, vol. 28, no. 4, p. 292, 2008.

[158] Y. C. Guo, "Lag syncope cannot be ignored," Shanghai Journal of Acupuncture and Moxibustion, vol. 14, no. 6, article 254, 1995. 
[159] Y. Z. Liu, "Analysis on two cases about abnormal condition induced by improper position during acupuncture," Lishizhen Medicine and Materia Medica Research, vol. 18, no. 7, p. 1756, 2007.

[160] Y. Liao and Y. Q. Guo, "Report of one case about syncope induced by acupuncture," Journal of Clinical Acupuncture and Moxibustion, vol. 25, no. 8, article 21, 2009.

[161] J. P. Zhang, "Disinfection must be done when needling in ExUE19," Chinese Journal for Clinicians, vol. 2, article 14, 1980.

[162] C. Z. Xu, "One case about intracranial infection caused by acupuncture," Nei Mongol Journal of Traditional Chinese Medicine, no. 1, p. 12, 1990. 


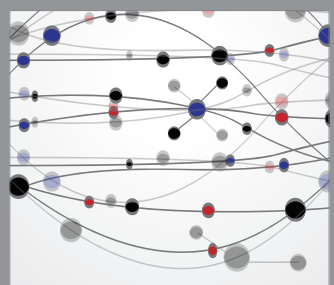

The Scientific World Journal
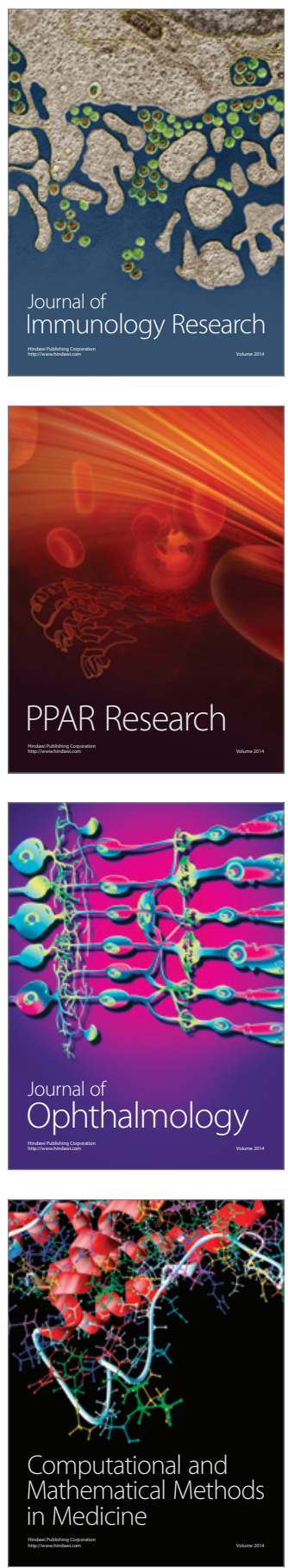

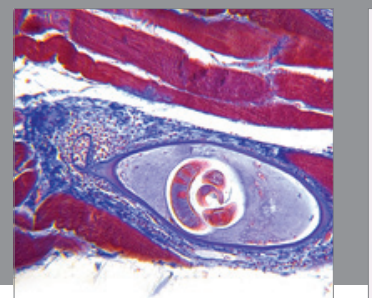

Gastroenterology

Research and Practice
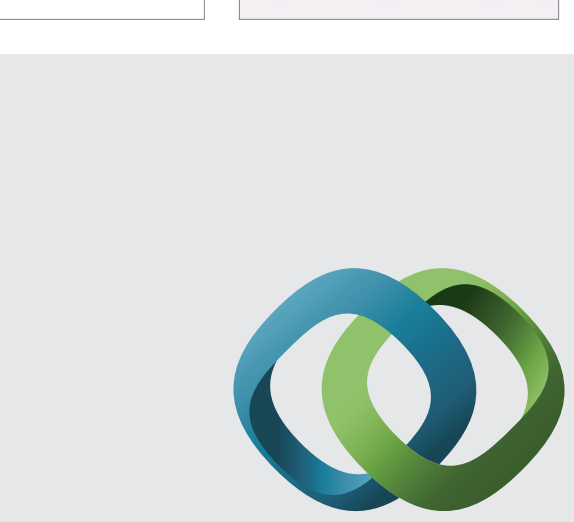

\section{Hindawi}

Submit your manuscripts at

http://www.hindawi.com
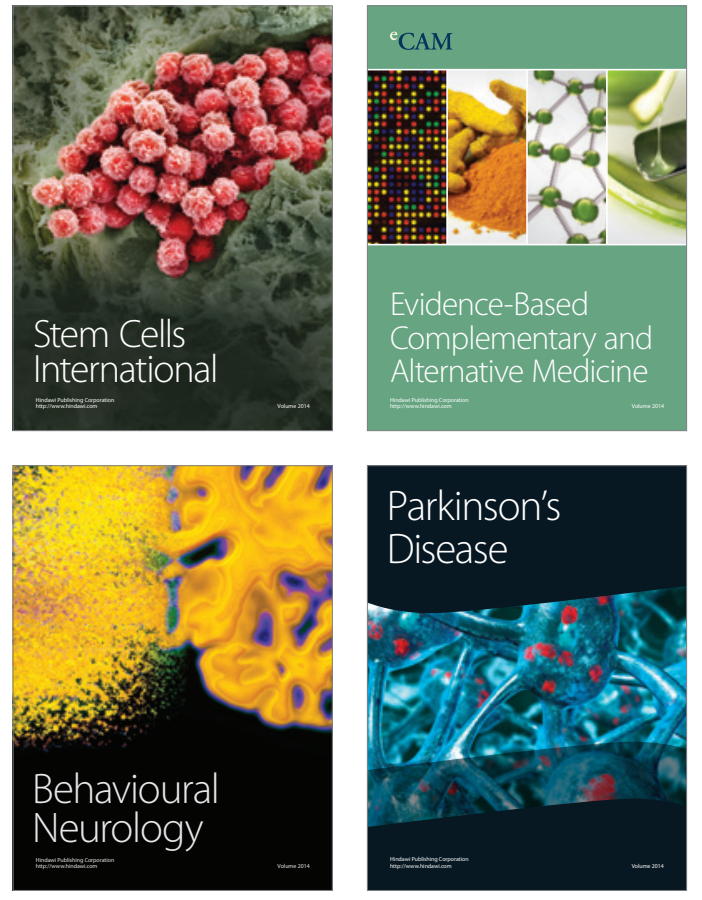
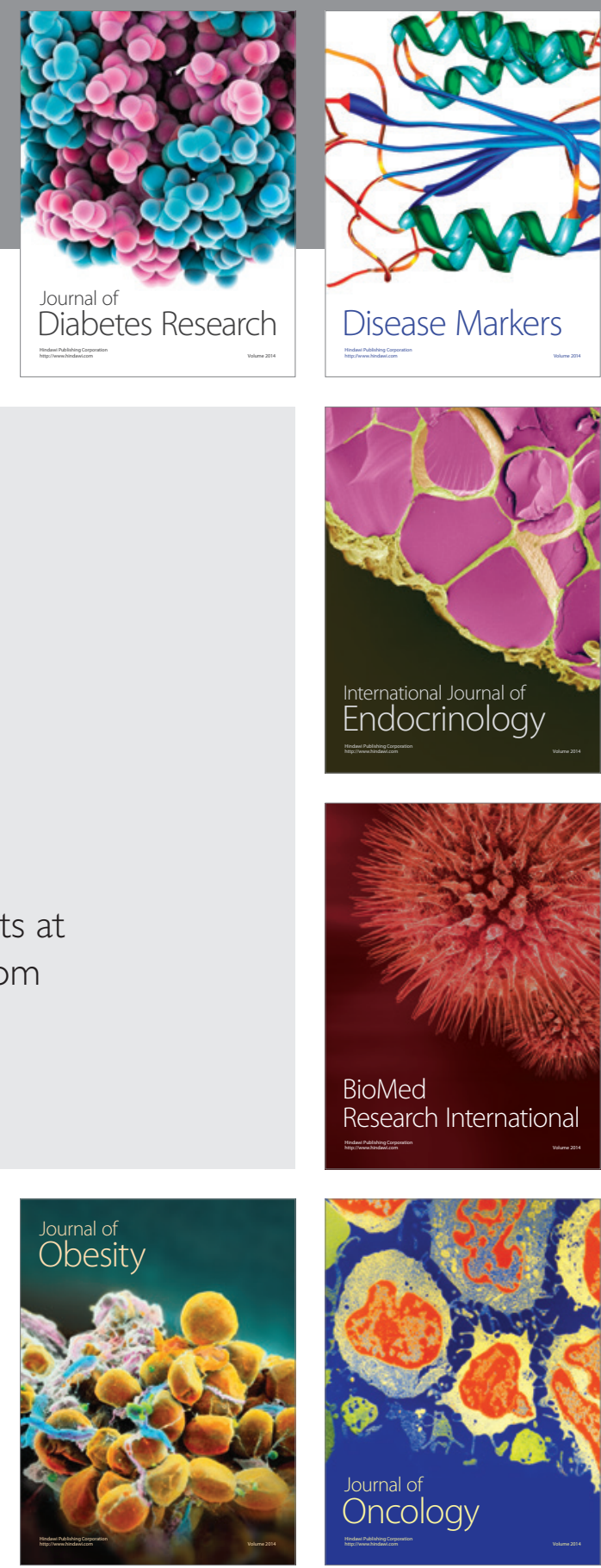

Disease Markers
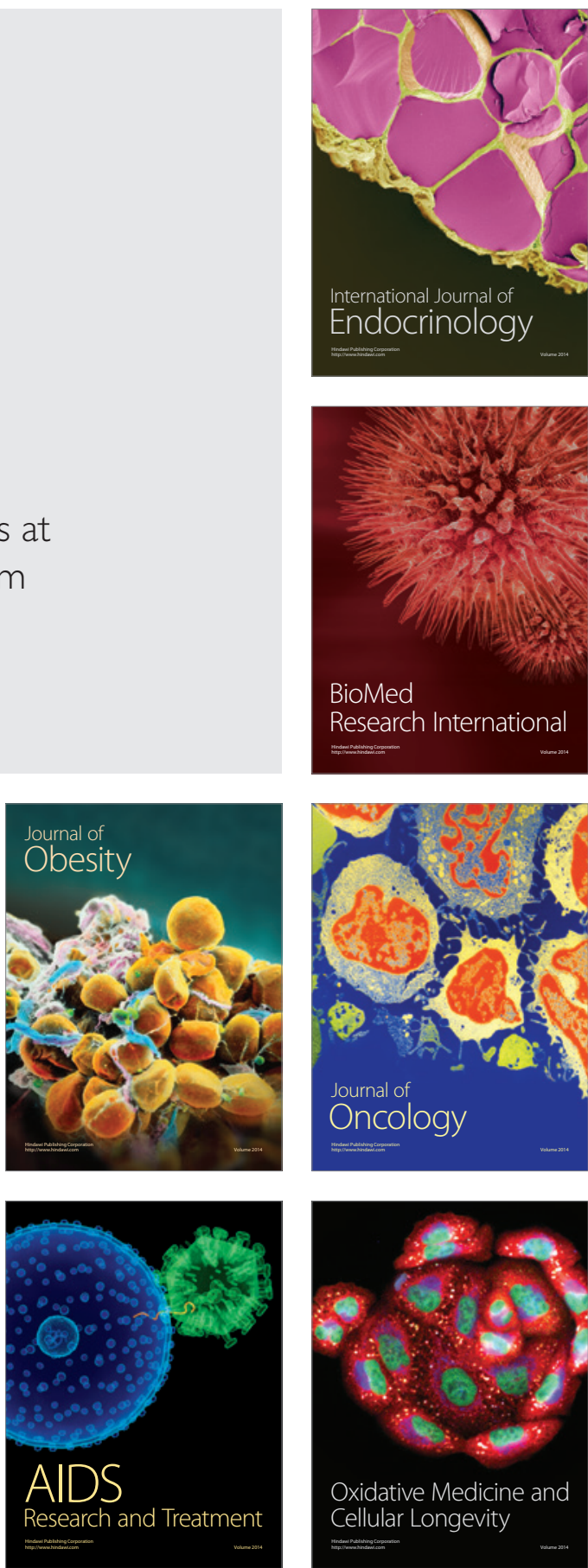